\title{
ABSTRACTS: WFSICCM/WFCCN World Congress 28 August - 01 September 2013, Durban, South
}

\section{Africa}

\section{World Federation of Critical Care Nurses}

Key words: abstracts * conference critical care nursing * WFCCN

Website: www.wfccn.org

\section{INTRODUCTION}

The 11th Congress of the World Federation of Societies of Intensive and Critical Care Medicine (WFSICCM), entitled Critical Care For All, was held in Durban, South Africa between 28 August to 01 September 2013. It was hosted by the Critical Care Society of Southern Africa, in conjunction with the World Federation of Pediatric Intensive and Critical Care Societies (WFPICCS), and the World Federation of Critical Care Nurses (WFCCN).

All nursing free papers were selected following peer review.

\section{ABSTRACTS}

Infection development in patients hospitalized in the intensive care unit following chest surgery

Besey Ören (1), Isik Kaymak (2), Neriman Zengin 91), Gülcin Bozkurt (1), Saadet Yazici (1)

(1) Istanbul University, Health Science Faculty, Department of Midwifery, Istanbul, Turkey; (2) Istanbul University Cerrahpasa Medical Faculty, Department of Thoracic Surgery, Istanbul, Turkey

Introduction: Patients may develop infections as a result of being hospitalized in intensive care and they may also need intensive care because of infections.

Objectives: To investigate infection development in intensive care patients following chest surgery.

Methods: Data for this descriptive and cross sectional study was obtained by examining patient files who were hospitalized during 2007-2010 in a Surgical Intensive Care Unit following chest surgery. A 25-item questionnaire was used. Data was analyzed using the SPSS 15. Chi-square, independent groups t-tests, and ANOVA were conducted.

Results: The mean age was $56 \pm 16.23$ (18-81). Among the patients, $67.8 \%$ were male, $72 \%$ were married, $45.2 \%$ graduated from primary school, $65.6 \%$ smoked, and $51.6 \%$ had an additional condition. $66.9 \%$ were diagnosed with lung cancer and operated. Reasons for being hospitalized were post-operational follow-up in $63.5 \%$ of the patients and acute respiratory failure in $15.6 \% .49 .5 \%$ stayed in intensive care for one day, $10.8 \%$ for two days, and $6.5 \%$ for four days. $17.2 \%$ developed infections during their stay in intensive care. The predominant infection factors were stafilococcus aurous $(4.4 \%)$, MRSA (3.3\%), and pneumococcus $(2.2 \%)$
There was no relationship between infection development and age, gender, educational status, smoking, and having an additional condition $(p>0.05)$. There was a significant relation between infection development and the duration of stay in intensive care $(p>0.05)$.

Conclusions: The rate of infections increase as the duration of stay in intensive care increases. Discharging patients from intensive care as soon as their acute status improves may decrease infection rates.

Patterns of behaviour and interaction in the critical care unit

Suegnet Scholtz, Elzabe Nel, Marie Poggenpoel, Chris Myburgh University of Johannesburg, Johannesburg, Gauteng, South Africa Introduction: The well-being of Critical Care nurses is of great concern. One of the strategies posed for the chronic shortage of well-trained health workers by the World Health Organization (2006:67) is: "helping the existing workforce to perform better." In order to meet the needs of their patients or in other words; 'perform better', Critical Care nurses should be whole themselves. These nurses have adapted to the fast-paced and stressful environment of the critical care unit, by functioning within their own, unique culture. Studying this culture provides the key to understanding and the basis for facilitating wholeness in Critical Care nurses.

Objectives: To explore and describe the culture of critical care nurses in a critical care unit.

Methods: An explorative approach with an ethnographic case study design was used. Thirteen registered nurses participated in this research. A process of data-triangulation included data from field notes of participant observation, participant interviews and diaries. Data was coded and further analysis resulted in discernible patterns.

Results: Patterns of patient adoption, patterns of armour display, patterns of despondency because of the demands to adjust, patterns of "sibling-like" teamwork and patterns of non-support from management and the medical (physicians and surgeons) team constitutes the culture of Critical Care nurses.

Conclusions: The Critical Care culture is identified by specific patterns of behavior and interaction between the individual, team and external Critical Care environment.

References: World Health Organization. The world health report 2006: working together for health. WHO press, Geneva. Available from: http://worldhealthreport_2006.pdf (Accessed 17 October 2007). 


\section{Psychological problems in burn patients}

Nebahat Yildiz (1), Aysel Gürkan (2), Imren Avsar (1), Ayse Hale Uysal (1), Besey Ören (3), Saadet Yazici (1), Gulbahar Keskin (1)

(1) Istanbul University, Istanbul Faculty of Medicine, Department of Surgery, Istanbul, Turkey; (2) Marmara University, Health Science Faculty, Department of Nursing, Istanbul, Turkey; (3) Istanbul University, Health Science Faculty, Department of Midwifery, Istanbul, Turkey.

Introduction: Burnt patients cause an increase in the mortality and increase in the psychological problems that we have to face.

Objectives: In this study our goal is to assess the incidence of the psychological problems of the burnt patients after burn.

Methods: A retrospective, defining study of all patients ( $N=448)$ admitted Burn Unit from October 2004 through December 2008. Patients with psychological problems after burn were enrolled to Group $1(n=63)$ and the patients without psychological problems were enrolled to Group $2(n=385)$. Statistical analyses were performed by SPSS. An adjusted $p$ value $<0,05$ was considered statistically significant. Values are reported as median, mean, standard deviation for continuous variables and $\times 2$ test, t-test, Pearson Corelation technique were used to evaluate the values.

Results: 69 of the 448 patients who administered to the burn unit had got psychiatry consultation, it was figured out that there were psychological problems in 63 patients after burn and in 6 patients before burn. There were significant differences between the patients' ages, burn localization, burn cause, percentage, severity, existance of an infection, surgical approach, intensive care experience, intensive care unit and hospital length of stay. There were significant differences between age, cause of burn $(p<0,001)$ and the place where the incident happened $(p<0,001)$ in both groups. It was showed that surgical approach, intensive care experience cause higher incidence in psychological problems after burn $(p<0,001)$.

Conclusions: These study it is showed that surgical approach, intensive care experience are important factors which cause psychological problems after burn.

References: 1. Çakır S, Kulaksızoğlu IB. Psychological support of burn Injury patients and burn unit staff. Turkiye Klinikleri J Surg Med Sci 2007; 3(1):116-20. 2. Kidal M, Willebrand M, Anderson G, Gerdin B, Ekselius L. Personality characteristics and perceived health problems after burn injury. J Burn Care Rehabil 2004; 25:283-35. 3. Steenkamp WC, Albertyn R. Psychosocial factors that influence the outcome of burn treatment. CME 2008; 26(9):424-26. 4. Levenson $\mathrm{JL}$. Psychiatric disorders among burn patients. Psychiatry Weekly 2007; 2(29):5-6. 5. Van Loey NEE, Van Son MJM. Psychopathology and psychological problems in patients with burn scars. Am J Clin Dermatol 2003; 4(4):245-72.

The effects of simulation training based on mastery learning model on advanced cardiac life support acquisition

\section{Eunok Kwon}

\section{Seoul National University Hospital, Seoul, Republic of Korea}

Purpose: The Purpose of this study was to examine the effects of simulation training based on mastery learning model regarding Advanced Cardiac Life Support(ACLS) acquisition.

Methods: A quasi-experimental study was carried out with a nonequivalent control group pre-post test design. Participants were recruited 77 critical care nurses, who had worked in the intensive care unit of one tertiary hospital in Seoul from June 7th to June 10th 2011. Moreover, one experimental and three control groups were consisted of 18 21 nurses each. The experimental and control 2 group had the simulation training based on mastery model. However, control 1 group received the traditional education without simulation training based on mastery model and control 3 group had none. After the 2-hour simulation training on mastery learning, participants completed a post-test measuring knowledge, self efficacy, and clinical performance ability. The SPSS WIN 17.0 program was applied to analyze the collected data.

Results: The experimental group had a higher mean score for performance ability than control groups significantly. However, the experimental and control groups did not differ significantly in the level of knowledge and self-efficacy.

Conclusions: The simulation training based on mastery learning is an effective nursing education program for improving the clinical performance ability of ACLS.

Fluid balance monitoring in critically ill patients

\section{Annette Diacon}

University of Stellenbosch, Stellenbosch, South Africa

Introduction: Accurate fluid balance monitoring results in better outcome for patients, a positive fluid balance may predict higher mortality in critically ill patients (1). An informal audit performed in a local critical care unit, 7 out of 10 fluid balances were incorrectly calculated.

Objectives: The aim was to describe perspectives and practices of registered nurses in critical care units with regard to fluid balance monitoring.

Methods: A quantitative approach in the form of an audit established the current practice of fluid balance monitoring. A survey was conducted among registered nurses to gain insight into their perspectives of fluid balance monitoring. A purpose designed audit tool and questionnaire was used. The Mann-Whitney-U-test tested correlation.

Results: The audit revealed that $90 \%$ of the fluid balance records were inaccurately totalled (tolerated deviation $0-10 \mathrm{ml}$ ). Greater calculation inaccuracy of fluid balances correlated positively with patients receiving diuretics. Survey data showed registered nurses consider correct fluid balance monitoring as an important part of patient-care and are aware that inaccurate calculations can pose a risk to patients.

Conclusions: The study results are similar to other internationally studies. Nurses recognise the risks of fluid balance calculation errors. The audit data demonstrates that fluid balance calculation practices require practical solutions to support safe patient-care.

References: (1). Vincent JL, et al. Sepsis in European ICU: Results of the SOAP study. Critical Care Medicine 2006;34,2:344-353.

Failure mode effect analysis on administration of 1:1 concentrated potassium chloride (KCL) in a children's intensive care unit (CICU) at Kandang Kerbau Women's and Children's Hospital.

Yoke Kuan Kong, Nguk Lan Pang, Siew Kum Lee, Yoke Hwee Chan, Ang Noi Lee, Su Ling Ang, Meow Kui Seow, Siew Cheng Neo

KK Women's and Children's Hospital, Singapore, Singapore

Introduction: $\mathrm{CICU}$ practices administration of concentrated $\mathrm{KCL}$ 1:1 dilution for cardiac patients for correction of low serum potassium. Chief Pharmacist had a concern with practices of administering 1:1 KCL dilution and had raised this issue to Patient Safety council.

Objectives: Standardized ordering of concentrated KCL infusions in Closed Loop Medication Management (CLMM) system and formulated guidelines to ensure measures were adequate to prevent the risk of undesirable outcomes. 
Methods: Our group analyzed the entire administration process of intravenous KCL infusion. We identified potential failures mode that impact on patient safety in the various sub-processes that required improvements. Using the FMEA scoring system, it was clearly indicated that the prescription and administration processes have the potential for hazardous errors. The group listed out appropriate safety measures to prevent potential medication errors pertaining to these two processes.

Results: Post implementation of the safety measures, reassessment of the prescription and administration processes had shown significant reduction in risk priority number scores which indicate the success of FMEA in minimizing patients' harm.

Conclusions: Standardizing $\mathrm{KCL}$ infusion guideline and built-in safety features in CLMM can prevent medication error, assuring better patient safety and preventing sentinel events. It also creates an environment of safe practice for the staff. This will ultimately increase satisfaction of nurses in their daily work which reduces nursing turnover and make care more efficient.

Effectiveness of educational intervention on initiation of nursing-led feeding protocols on the adoption of nursing preferred teaching methods: a questionnaire survey

\section{Ang Noi Lee}

KK Women's and Children's Hospital, Singapore, Singapore

Introduction: Children in the intensive care unit are at risk of malnourishment. Nurse-led feeding protocol has proven to be effective in attaining nutritional goals in the paediatric intensive care unit (PICU. We hypothesized that understanding of preferred teaching methods are essential to help enhance effectiveness of teaching prior to introduction of a new feeding protocol in the PICU.

Objective: To assess the effectiveness of an educational intervention on initiation of nursing-led feeding protocols based on nursing preferred teaching methods.

Methods: A follow-up questionnaire was posted to all nurses in $\mathrm{CICU}$ where specific training had been provided according to nursing preferred teaching methods to assess the effectiveness of the sessions and nurses comfort level with the use of new feeding protocol.

Results: Fifty-two nurses completed the questionnaire survey. The majority $(80 \%)$ agreed that the teaching was useful. Of the 53 respondents who received the feeding protocol manual, $32(86 \%)$ agreed that the protocol was useful to them. Overall, $79 \%$ of the respondents thought that the different teaching methods were useful. However there were about $14 \%$ who attended the teaching sessions strongly disagree that different teaching sessions were useful. In general, majority (total: about $70 \%$ ) were relatively comfortable in using the protocol after the teaching sessions.

Conclusions: Understanding preferred teaching methods has proven to help enhance effectiveness of teaching prior to introduction of a new protocol in the PICU.

References: Neef M et. al. Nutritional goals, prescription and delivery in a pediatric intensive care unit. Clinical Nutrition 2008; 27: 65-71

Intensive care unit nurses' current problems and expectations

\section{Meral Madenoglu Kivanc}

Istanbul University Faculty of Health Sciences, Istanbul, Turkey

Aim: to reveal intensive care unit nurses' problems and expectations regarding their work, health, communication, education and management.

Methods: This is a descriptive study performed between September and December 2011 on 207 nurses working in intensive care units of hospitals in Istanbul. Data were collected with a questionnaire including 40 questions and analyzed with NCSS 2007 and descriptive statistics.

Results: Of 207 nurses, $38.6 \%$ were aged $26-30$ years, $58 \%$ were university graduates, $68.1 \%$ had $1-5$-year-experience in ICUs and $78 \%$ were working for $51-60$ hours weekly. $73.9 \%$ fulfilled physicians' duties and $67.1 \%$ fulfilled secretarial duties besides their own work, $57 \%$ believed that there is cooperation between nurses and physicians and $45 \%$ believed that there is cooperation between nurses. $57.5 \%$ claimed that they do not have work safety and $72.4 \%$ of them most frequently had backache. $52.1 \%$ demanded that ICU nurses should be offered further education following university. $39.6 \%$ noted that they were exposed to autocratic management, but $39.1 \%$ reported that managers appreciated their performance. Causes of leaving their jobs were an insufficient number of staff mentioned by $48.7 \%$ of the participants, long working hours mentioned by $43.9 \%$ of the participants and low income mentioned by $31.8 \%$ of the participants.

Conclusions: Weekly working hours should be reduced, job descriptions should be made properly, income and the number of staff should be increased, cooperation between team members should be improved, the democratic management style should be adopted and work safety should be guaranteed so that intensive care nurses can work effectively and efficiently.

The knowledge and application of nurses regarding pain management of intubated intensive care patients

Meryem Turkoglu (1, 2), Neriman Akyolcu (2), Mehmet Isik (1)

(1) Sisli Etfal Teaching and Research Hospital, Istanbul, Turkey; (2) Halic University, Istanbul, Turkey

Introduction: This study was applied as a descriptive research between the dates 28/11/2012-28/02/2012 in order to define the information and practices of the nurses about the pain control of the intubated intensive care patients and bring forward some suggestions about the pain control of the intubated patients.

Methods: The sample is consisted of 87 nurses who accept to join the study. The data of the research was brought together by a survey that contains the information and treatments of the nurses regarding their pain evaluation. The data of the research was analyzed by using the program SPSS 17.0

Results: It was determined that $65 \%$ of the nurses are informed about pain management, $35,6 \%$ of them define the pain as "a condition which arouses discomfort and an unpleasant situation", $68 \%$ of the nurses use pain evaluation and monitoring form, $96,6 \%$ describe the surgical interventions as the cause of pain, $56,3 \%$ of the nurses define tachycardia as the physiological sypmtom of the pain, $46 \%$ of them define agitation as the behavioral pain symptoms, $34,5 \%$ of them use pain evaluation and monitoring form on intubated patients; $73,3 \%$ of the nurses who use form prefer facial expression scala,the average point of information and treatment regarding pain evaluation is at a level of $18,494 \pm 3,091$, the points of information and treatment regarding pain evaluation of the female nurses $(x=18,833)$ is significantly higher $(p=0,020<0,05)$ than the male nurses' points $(x=17,429)$ the points of the nurses who use monitoring form are lower than nurses who do not use monitoring form $(x=17,900)$ and the difference between is significant $(p=0,009<0,05)$.

Development of clinical learning supervision in the neurointensive care unit Helsinki, Finland

\section{Sofia Hiekkanen, Elina Ristola}

Helsinki University Central Hospital, Helsinki, Finland

Introduction: The need for high quality tutoring in clinical practise is important in order to recruit professional new nurses for the field. Here we enlighten how clinical practise of the nursing students has 
been improved in the Neurointensive care ward (NICU) in Helsinki University Central Hospital (HUCH).

Objectives: Clinical practice has been under development in NICU recently. The need for further development is based on experience, the KASTE programme [1] and HUCH's strategic objective which enforces high level research and teaching. Goals concerning health care in the KASTE programme are to develop education, to ensure know-how and to target human resources efficiently.

Methods: In NICU two nurses are in charge of improving clinical practice. They attended a course in how to be a good instructor for students. Since then they have worked on making NICU the best learning environment possible. For example the structure and schedule for clinical practise has been updated, also the tutoring of students is now better organized and more methodical.

Results: CLES [2] has been created to evaluate how students experience their clinical learning environment and supervision given by staff nurses. In 2007 NICU obtained 6,77 (scale 1-10) CLES points and in 2012 9.20. Results show that the work of the nurses in charge of improving clinical practise has been worth the effort.

Conclusions: There are many future challenges regarding students. They include keeping the level of tutoring high and motivating new tutors.

References: [1] http://www.stm.fi/c/document_library/get_fi le?folderld=28707\&name=DLFE-3683.pdf\&title=Sosiaali_ ja_terveydenhuollon_kansallinen_kehittamisohjelma_ KASSTE_2008_2011_fi.pdf, [2] Clinical learning environment and supervision. Mikko Saarikoski. 2002. Turku.

Critical care nurses knowledge on adult mechanical ventilation

\section{Ram Sharan Mehta}

B.P. Koirala Instutte of Health Sciences, Dharan, Nepal

Introduction: Critical care nursing is that specialty within nursing that deals specifically with human responses to life-threatening problems.

Objectives: The objective of this study was to find out the knowledge on adult mechanical ventilation management among the nurses working in Intensive care unit of B.P. Koirala Institute of Heath Sciences.

Methods: It was hospital based descriptive study conducted among all the 35 nurses working in critical care unit of BPKIHS having work experiences at least 3 months in the same unit. Using pre-tested questionnaire having 50 items of knowledge, the data was collected during the period of 1-7-2010 to 7-7-2010 for a week maintaining all the formalities.

Results: Most of the nurses had adequate knowledge on the items like, definition of mechanical ventilation $(100 \%)$, care of tracheotomy $(88.6 \%)$, where as the limited nurses had knowledge on indication of CPR (25.7\%)and indication of continuous positive air-way pressure (CPAP) $(5.7 \%)$. The association calculated with items of knowledge score and other variables, it was found significant association between indication of non-invasive mechanical ventilation (MV) and training institute $(p=0.034)$, LMA and ICU experiences $(p=0.047)$, synchronized intermediate mandatory ventilation (SIMV) use and ICU experiences $(p=0.042)$ and goal of tracheostomy care and ICU experiences $(p=0.046)$.

Conclusions: Most of the nurses had average (score-1,2) knowledge on common knowledge components and very limited nurses had knowledge on the components that was not performed in our setting or very less frequently performed like, use of LMA, pacing and CPAP. Regular CNE is mandatory for the nurses working in ICU.
Critical care nurses skill working in B.P. Koirala Institute of Health Sciences Nepal

\section{Ram Sharan Mehta}

BP Koirala Instutte of Health Sciences, Dharan, Nepal

Introduction: BP Koirala Institute of Health Sciences has 700 bedded tertiary care hospital having 8 beded ICU and 6 beded CCU with modern facilities.

Objectives: The objective of this study was to assess the skills to care the critically ill patients by the nurses working in critical care unit (ICU and CCU) of B P Koirala Institute of Health Sciences, Dharan, Nepal.

Methods: It was hospital based descriptive study conducted among the nurses (staff-nurses) involved in the care of critical patients working in ICU and CCU of B. P. Koirala Institute of Health Sciences for more than three months. Using total enumerative sampling technique all the 35 nurses working in ICU and CCU of BPKIHS was selected for study. Informed verbal consent was obtained prior to data collection. Subjects were assured about the confidentiality of the Informations they were provided and used for the study purpose only. Using pre-tested questionnaire the data was collected during the period of 1-7-2010 to 7-7-2010 for a week.

Results: Out of total 35 nurses only 2 nurses $(5.7 \%)$ had received basic life support (BLS) and advanced life support (ALS) training. The association calculated between the items of skill score $(0,1$, $2,3)$ with selected variables found significant association between interpret arrhythmia and age $(\leq 20,>20)(p=0.0001)$, perform defibrillation and age $(p=0.002)$, and interpret arterial blood gas $(A B G)$ and age group $(p=0.016)$.

Conclusions: skill development training for nurses working in ICU is mandatory for quality care.

Knowledge and attitudes of nurses regarding pain management of ICU patients in three referral hospital in Rwanda

Christine Ufashingabire (1), Etienne Nsereko (1), Petra Brysiewicz (2)

(1) Kigali Health Institute, Kigali, Rwanda; (2) University of KwaZulu Natal, Durban, South Africa

Introduction: Pain is a significant burden experienced by almost all the patients admitted to the Intensive Care Unit (ICU).

Objectives: The aim of the current study is to describe nurses' knowledge and attitudes regarding pain management of ICU patients in three referral hospitals in Rwanda.

Methods: A cross-sectional survey was conducted. The population is comprised of all the nurses working in ICUs in the three referral hospitals. Data collection was carried out using a standardized questionnaire developed by McCaffery and Ferrell (2008). Analysis was carried out using the Statistical Package for Social Sciences SPSS, version 18. One single mean out of the total score was used to test if the hospital and a nurse's level of education have a significant impact on the importance given to potential barriers to pain management.

Results: The study shows that participants are young adults and mostly female. The majority were A 1 level nurses. With regard to the participants' knowledge and attitudes towards pain management, the study revealed that nurses working in ICUs have knowledge a deficit and poor attitudes towards pain management. The study reported here also showed that working in high standards institution have a significant influence on the ability of nurses to appreciate barriers to pain management $(P<.0001)$. In addition, the results indicate that level of education has significant influence on the ability of nurses to recognize potential barriers to optimal pain management $(p<.008)$. 
Conclusions: The current study reveals that knowledge deficit and poor attitudes towards pain management are obvious among nurses

\section{A survey on ICU nurse's knowledge of sepsis}

\section{Sun Gyoung $\mathrm{Na}$ (1), Young Hee Yi (2)}

(1) Samsung Medical Center, Seoul, Republic of Korea; (2) Department of Clinical Nursing Science, Samsung Medical Center, seoul, Republic of Korea

Introduction: the ICU is where sepsis patients are usually treated, and has a high risk of sepsis outbreak due to invasive treatment and other reasons, so the role of nurses in the forefront of patient care is important.

Objectives: This study was conducted to determine the extent of knowledge on sepsis by ICU nurses and provide basic data for the provision of early intervention to patients with sepsis.

Methods: Data were collected using a structured questionnaire by nurses working in ICU for adult patients of a hospital in Seoul from September 10, 2012 to September 17, 2012. The questionnaire was composed of 30 items based on Robson et al. (2007)'s research tool and references to guidelines published by Dellinger et al. (2008).

Results: $89.1 \%$ of the participants wanted a sepsis screening tool. The therapeutic area of sepsis $(77.0 \%)$ was lower than areas of symptoms and signs (89.4\%), definition (82.4\%) and judgments about the cases (81.8\%). Knowledge about sepsis according to the general characteristics and sepsis-related experience score was significantly higher for those who were 25 years of age or older, had more than three years of clinical experience, had more than three years of ICU experience, was working in the MICU, and had nursing experience on patients with sepsis or had educated experience of sepsis.

Conclusions: It is suggested that educational programs be developed and applied to compensate the inadequacy, and to develop early screening tools that can be applied in clinics based on the results of this study.

Changing the practice of 'Chinese whispers': an action research approach to optimise the PICU nursing shift handover

\section{Clare Davis, Minette Coetzee}

The Child Nurse Practice Development Initiative, The University of Cape Town, Cape Town, South Africa

Introduction: Described as being similar to a "chain of Chinese whispers" (1), handover of patient information between health professionals is internationally recognised as a necessary but potentially risky aspect of patient care. Consequently, the World Health Organisation has called for a standardised handover approach. Effecting a change in healthcare has been identified as a challenging task; an increased use of participative change management methodologies has emerged as a result.

Objectives: The study aim was to optimise the quality and efficiency of nursing shift handover in the PICU at Red Cross Children's Hospital by use of a participative approach.

Methods: An action research approach was applied. Nurses from the study setting, together with the researcher, formed a core participant group. Details of the action research cycles conducted within the negotiation, assessment, planning and action stages will be presented.

Results: PICU nursing shift handover consists of a bedside and unit handover. Critique of the bedside handover highlighted challenges of content and process. This led to a handover form and Standard Operating Procedure being introduced. Critique of the unit handover emphasized difficulties experienced with time, attendance and irregular occurrence thus an additional scrolling PowerPoint handover was initiated.

Conclusions: PICU nursing shift handover was optimized and the chosen methodological approach facilitated achievement of this.

References: (1) Cleland, J. A et al, R. 2009. "There is a chain of Chinese whispers ...": empirical data support the call to formally teach handover to prequalification doctors. Qual Safe in Health Care. 18(4): 267-71.

Development and application of the sleeve-type restraints for ICU patients

\section{Hyun-Young Ko (1), Jiyeon Kang (2)}

(1) Dong-A University Medical Center, Busan, Republic of Korea; (2) Dong-A University, Busan, Republic of Korea

Introduction: We have developed sleeve-type restraints to reduce the side effects of conventional wrist restraints. Two long sleeves are attached to the new restraints, and there is no knot tying to the patient's body.

Objectives: To compare the sleeve-type and wrist restraints.

Methods: Forty four pairs of ICU patients and their families participated in the experiment. The nurses applied sleeve-type restraints to the patients in the experimental group, and wrist restraints to the control group. The trained research assistants measured ROMs, skin temperature, edema, and skin lesions of both upper extremities (UEs) before, 24 hours, 48 hours, and 72 hours after the restraints applied. The emotional response of family was measured 72 hours after the restraints applied. Thirty one ICU nurses evaluated the application efficiency for both types of restraints.

Results: Compared to the control group, changes of ROMs, edema, and skin abrasions on both $\mathrm{U} /$ Es of the experimental group indicated a significant difference in physical side effects. However, there were no differences in occurrence of redness and skin temperature in the experimental group as compared to the control group. The emotional response scores of the experimental group were significantly lower than those of the control group. The mean scores of application efficiency for the sleeve-type restraints were significantly higher than those for the wrist restraints.

Conclusions: The results of this study indicate that the sleeve-type restraints are better than the conventional wrist restraints with respect to physical side effects, emotional responses of family members, and application efficiency.

ICU nurses' perception of work environment and horizontal violence

\section{Jiyeon Kang}

Dong-A University, Busan, Republic of Korea

Introduction: The nursing shortage has been emerged as a social problem in Korea. Many nurses decide to leave their jobs because of poor working environment, and interpersonal relationships are main elements that make up the nursing work environment.

Objectives: This study was aimed to investigate the relationships between the perception of nursing work environment and horizontal violence among nurses in the intensive care unit (ICU).

Methods: This is a descriptive survey research, with 134 ICU nurses from 5 different hospitals. The work environment was measures by the Korean nursing work environment scale. The horizontal violence was measured with the Korean version of negative acts questionnaire-revised (NAQ-R) measuring workplace bullying and the horizontal violence scale measuring nurse to nurse violence. 
Results: ICU nurses reported moderate satisfaction with their work environment, with perception of the basic work system receiving the highest scores. $94.0 \%$ of ICU nurses have experienced horizontal violence within the past 6 months, and the prevalence of bullying was $17.7 \%$ according to operational bullying criteria. ICU nurses report they experience more work-related violence than other types of violence. Significant negative correlations between work environment and horizontal violence were found.

Conclusions: These findings suggest that the better the nursing work environment, nurses will experience less horizontal violence. Further research needs to be done to develop an appropriate instrument to assess the horizontal violence among nurses and to build a multidimensional model of horizontal violence in the nursing workplace.

Factors leading to non-divulgence by patients who use traditional medicine in Westrand Mine critical care units

\section{Sidwell Matlala, E Nel, MM Chabeli}

University of Johannesburg, Johannesburg, South Africa

Introduction: The majority of Africans use traditional medicine, even those who have easy access to modern medicine and can afford it; they still prefer to visit traditional healers. Those visits are often surrounded by an atmosphere of secrecy (Carrera, 2006). The researcher undertook the study about "factors leading to nondivulgence by patients who use traditional medicines in the Westrand mine critical care units".

Objectives: The purpose of the study was to explore and describe factors leading to non-divulgence by patients who used traditional medicines in order to provide a comprehensive approach to care in Westrand Mine Hospital critical care units.

Methods: The researcher used qualitative, contextual, exploratory, descriptive research design. A phenomenological approach was used. The population was all the patients who were envisaged to have used traditional medicines in the Westrand Mine hospital, and did not divulge (Mouton, 2010).

Results: 12 participants were purposively selected from a hospital register, these patients displayed symptoms related to use of traditional medicine, and the conditions identified from the register were as follow: kidney failure, liver failure, gastro-enteritis, and headache.Semi-structured individual interviews were conducted. Ethical principles were adhered to, and trustworthiness was ensured (Burns \& Grove, 2009). Qualitative open coding method of data analysis was used according to Tesch's protocol (Creswell, 2013). The following theme and subthemes emerged from the data analysis: Fear and anxiety (main theme). Subthemes: fear to divulge secrets, fear of negative attitudes from nurses and fear to loose norms and values. The researcher will use the theme, subthemes and literature to formulate strategies to facilitate divulgence.

Training and 24-hour support from a mobile intensive care nurse: nurse-to-nurse collaboration in a university hospital in Norway.

Siv K Stafseth (1, 2), Sturle Grønbeck (1), Irene Randen (3), Tine Lien (3), Anners Lerdal $(4,5)$

(1) Department of Emergencies and Critical Care, Oslo university hospital-Rikshospital, Oslo, Norway; (2) Department of Research and Development, Oslo university hospital-Ullevål, Faculty of Medicine, University of Oslo, Oslo, Norway; (3) Department of Master and Continuing Education in Nursing, Lovisenberg Diaconal University College, Oslo, Norway; (4) Department of Research, Lovisenberg Diaconal Hospital, Oslo, Norway; (5) Department of Nursing Science, Institute of Health and Society, Faculty of Medicine, University of Oslo, Oslo, Norway
Introduction: Over the past 10 years rapid response systems have been implemented in Australia, Europe, USA and South Africa. The Modified Early Warning Score (MEWS) is a tool for assessment and detection of failure in patients' vital functions. In our hospital, the Mobile Intensive Care Nurse (MIS) started an educational program for RNs and students in hospital wards. The program aimed to provide theoretical and practical MEWS training and to inform RNs of the availability of 24-hour MIS support. The MIS program is run solely by nurses as an "easy line for collaboration" between nurses.

Objectives: The objective was to explore RNs initial experiences using MEWS and MIS with patients-at-risk in two wards at Oslo University hospital in Norway. Study period: 6 months in 2011-12.

Methods: Mixed-methods exploratory study; survey and focus group interviews of RNs in hematology, gastrosurgical and urology wards. All 80 RNs completed the 4-hour education program and received questionnaires, and $48(60 \%)$ responded. Seven RNs with various levels of nursing experience volunteered for a focus group interview.

Results: The survey showed that $81 \%$ strongly agreed that the educational program improved their ability to detect patients at risk. Interviews were analyzed and three themes emerged: a) experiences with MIS and early recognition of patient deterioration and b) collaboration and transfer of knowledge and c) a "new" precise language with MEWS.

Conclusions: The educational program for MEWS and MIS was successfully implemented. RNs had good nurse-to-nurse collaboration and found a "new" language for communicating with physicians.

Long term cognitive Impairment and delirium in an Australian intensive care (LOGIC)

Marion Mitchell $(1,2)$, Leanne Aitken $(1,2)$, Bala Venkatesh (2), David Shum (3), Sharon Wetzig (2), Elizabeth Burmeister (2)

(1) NHMRC Centre of Reseach Excellence in Nursing, Centre for Health Practice Innovation, Griffith Health Institute, Griffith University, Queensland, Australia; (2) Princess Alexandra Hospital, Queensland, Australia; (3) Griffith University Health, Queensland, Australia

Introduction: Intensive care unit (ICU) patients have reported decreased cognitive function up to 2 years following their illness. Patients' delirium in ICU has been linked to poor outcomes including extended length of stay and reduced cognitive ability.

Objectives: To determine the incidence of delirium and cognitive dysfunction in Australian ICU patients.

Methods: Patients who were mechanically ventilated for $>12$ hours, able to give informed consent and available for followup, were recruited. The Repeatable Battery for the Assessment of Neuropsychological Status (RBANS) was conducted at 3 and 6 months. Patients were classified as being mildly to severely cognitively impaired, with education and age specific norms incorporated. Ethical approval was granted.

Results: Currently 44 participates have been recruited with 22 tested at 3 months and 9 at 6 months. Participants had a median age of 58.5 years (IQR: $37.2-71.0), 36(82 \%)$ males, median length of ICU stay 5.0 days (IQR: $3.0-9.0$ ) and mechanical ventilation 56.0 hours (IQR: 29.5-127.7). Eight patients (18\%) were delirious during their ICU stay. RBANS mean scores (Table 1) show improvement at 6 months.

Conclusions: Delirium rates in this cohort were low, however, its impact on cognition requires further study to assess long term patient outcomes. 
Table 1. RBANS mean scores (SD)

\begin{tabular}{|l|l|l|}
\hline & Time 1 $(\mathrm{n}=22)$ & Time 2 $(\mathrm{n}=9)$ \\
\hline Immediate Memory & $77.00(13.58)$ & $89.78(12.44)$ \\
\hline $\begin{array}{l}\text { Visuospatial/ } \\
\text { Construction }\end{array}$ & $103.50(18.42)$ & $103.78(18.99)$ \\
\hline Language & $93.91(14.90)$ & $99.67(5.70)$ \\
\hline Attention & $92.73(17.38)$ & $94.11(13.96)$ \\
\hline Delayed Memory & $84.00(17.51)$ & $93.33(13.91)$ \\
\hline Total RBANS & $86.91(13.37)$ & $94.44(13.51)$ \\
\hline
\end{tabular}

Early mobilization in intensive therapy (MIT-Project)

Anne Skafte, Anne Langvad, Maria Rothgart, Anna Bonderup, Suzanna Fragtrup, Tina Hartley, Ingrid Egerod

Slagelse Sygehus, Slagelse, Sjælland, Denmark

Introduction: Critically ill patients experience impaired functioning during and after hospitalization. Physical sees weakening as balance problems and decreased ability to walk distance, mentally as delirium and difficulty concentrating. The condition is associated with increased mortality, prolonged hospitalization and reduced selfrated health.

Objectives: Early systematic mobilization, improves critically ill patients' physical and mental functioning. MIT-project contributes with valuable knowledge about how soon and with what intensity, it is possible to train critical care patients through a training program.

Methods: Multicenter study with a prospective outcome in 35 patients ventilated more than 48 hours. Patients followed a training program with early intervenering, and compared to a historical control group of 58 patients who received conventional training.

Results: Preliminary compilation of data of the training program in clinical use shows that:

- Training of the patient begins earlier in the intervention group than in the control group.

- $\quad$ All exercises are possible in critically ill patients.

- $\quad$ Each training session items from between 15 - 45 minutes.

- Not all assigned exercises will be used during the training phase 2 and 3.

- $\quad$ Patients are often discharged before stage 5 and 6.

- The effect of exercise on physical and mental functioning is not yet determinable.

Conclusions: Training program contributes to that the critically ill patient is trained early. Training intensity must be organized, so that the critically ill patient either train in bed, out of bed or walking, in a training program which is reviewed in the light of the survey results.

Reference: Clinical Trials.gov Identifier: NCT01549808

Self-handicapping in intensive care nurses

\section{Sevim Ulupınar (1), Gülçin Bozkurt (2)}

(1) Marmara University Faculty of Health Sciences, Istanbul, Turkey; (2) İstanbul University Faculty of Health Sciences, Istanbul, Turkey

Introduction: Self-handicapping is the individual's tendency to externalize failure and internalize success; which is a behavioral habit displayed by the individual in order to reserve self-respect and confidence in the face of threatening situations.

Objectives: Our aim was to determine the factors affecting selfhandicapping in intensive care (IC) nurses.

Methods: The sample of the study consisted of 180 IC nurses. Data was collected with the questionnaire form and the Self-Handicapping
Scale. Data were analyzed using frequencies, means, correlations, ANOVA, and t-test.

Results: $89,4 \%$ of the nurses were female, $47,8 \%$ married, $87,2 \%$ nurses, $73,2 \%$ had Bachelor's degrees, $78,9 \%$ were satisfied with working in IC. The mean age was approximately 30 years, mean work experience was 7 years and mean work experience in IC was 5 years. $55 \%$ had high levels of job satisfaction; however, $77,8 \%$ reported to have a heavy workload. The mean self-handicapping score was 68,61 .

Conclusions: The level of self-handicapping decreased with age. Nurses who were vulnerable, were afraid of failure, had low selfesteem, had difficulty in making quick decisions, and were anxious displayed high levels of self-handicapping. Nurses who have good interpersonal relationships had low levels of self-handicapping. The level of self-handicapping did not show significant differences according to educational status, work and IC experience, and satisfaction with working in IC.

References: Akın A. Self-handicapping Scale: A study of Validity and Reliability. Education and Science 2012, 37(164),76-187; Akın A, Abacı R, Akın Ü. Self-handicapping: A Conceptual Analysis. International Online Journal of Educational Sciences, 2011, 3 (3), 1155-1168

Circadian types, sleep disturbances and medication errors in Korean rotating shift ICU nurses

Young Hee Yi (1), Su Jung Choi (2)

(1) Department of Clinical Nursing Science, Samsung Medical Center, Sungkyunkwan University School of Medicine, Seoul, Republic of Korea; (2) Samsung Medical Center, Seoul, Republic of Korea

Introduction: The nursing role is safety-critical and nurses are more frequently involved in medication errors than other health care professionals. Considering less sleep may increase the likelihood of medication errors, it is necessary to investigate sleep disturbances among rotating shift nurses working in intensive care units (ICUs) for ICU patients' safety.

Objectives: To identify circadian types, sleep disturbances, and medication errors in rotating shift ICU nurses and to analyze the correlation among three variables.

Methods: A cross-sectional descriptive survey was conducted. 137 nurses from 9 ICUs in a hospital were asked their circadian types using the Korean translation of composite scale (KtCS), sleep disturbances by insomnia severity index (ISI), and medication errors by a self-report questionnaire.

Results: Circadian types of the participants were 15 nurses $(10.9 \%)$ of the morning type, 106 nurses $(77.4 \%)$ of the intermediate type, and 16 nurses $(11.7 \%)$ of the evening type. 74 nurses $(54.0 \%)$ had sleep disturbances. 25 of 135 nurses (18.5\%) reported experienced medication errors for past 2 weeks. Work hours and sleep hours at night shift were significantly correlated with medication errors. The odds ratio for sleep hours at night shift and medication errors was 0.69 .

Conclusions: ICU nurses had sleep disturbances and less sleep increased medication errors. Developing strategies to reduce sleep disturbances of night shift nurses are needed particularly.

The profile of Istanbul University intensive care nurses

\section{Gülçin Bozkurt, Besey Ören, Neriman Zengin, Meral Madenoglu Kivanc}

Istanbul University Health Science Faculty, Istanbul, Turkey

Introduction: The recent changes in Turkey's health policies lead to rapid changes in the profile of intensive care (IC) nurses. 
Methods: This descriptive study, which aimed to examine the profile of Istanbul University IC nurses. The universe of the study consisted of 345 nurses who work in the Istanbul University IC. The sample included 202 nurses. Data was collected using the 22-question form and analyzed with the SPSS and evaluated with percentiles and mean tests.

Results: The mean age was $29,68 \pm 5.35$ years. $92 \%$ were nurses and $8 \%$ midwives/emergency medical technician and students. The majority had bachelor's degrees $(62,9 \%)$. The mean duration of working in intensive care was $5.59 \pm 5.26$ years. The mean duration of work/day was $11,30 \pm 1,70$ hours, and 41,52 $\pm 4,32$ hours per week. The nurse patient ratios were $1: 3$ in $54,5 \%$ of the sample and $1: 2$ in $28.7 \%$. The rate of working in IC immediately after graduation was $39.6 \%$, whereas the rate of nurses who received certificate education was $54.5 \%$. $70.3 \%$ of the nurses preferred to work in IC, $63.9 \%$ were satisfied with working in IC, $96.5 \%$ were underpaid, and $69.8 \%$ had health problems. $92 \%$ of the nurses stated that they did work beyond their responsibilities, such as secretarial work $(18,8 \%)$, everything but care $(14.4 \%)$, physician's responsibilities $(9.9 \%)$,

Conclusions: The majority of the nurses in our sample started to work in IC without receiving any education in this field. Most of them had to do work beyond their job descriptions, had health problems $(3 / 4)$ and were underpaid.

Exploring the needs of family members of patients in the intensive care unit of one hospital in Kigali, Rwanda

\section{Paul Munyiginya, Petra Brysiewicz}

Kigali Health Institute, Kigali, Rwanda

Introduction: The admission of patients in ICU is a stressful experience for the family members and there has been limited research adressing this issue in Kigali, Rwanda.

Objectives: The purpose of this research study was to to explore the needs of family members of patient's admitted into in ICU.

Methods: This study used a quantitative exploratory design focused on exploring the needs of family family members of patients in ICU at one hospital in Kigali, Rwanda. Forty family members were recruited using the convenience sampling strategy and completed the Critical Care Family Needs Inventory( CCFNI) (1).

Results: The needs for assurance was mentioned as very important need, followed by the need for comfort, the need for information, the need for proximity, and lastly the need for support. Respondents identified an additional three needs not previously mentioned in the the research tool which are to avail ressources for the patient, especially drugs so that the family members don't have to go to search for the prescribed drugs outside the hospital, respondents needed to have enough space in ICU to accommodate families members during visitation and to have a free space where to eat food near the ICU.

Conclusions: These results offer insights for nurses and other health care professionnals on how to appropriately care for the family members in ICU within a resource constrained environment.

Reference: (1). Molter, N.C. Needs of relatives of critically ill patients: A descriptive study. Heart Lung, 8:332-339.

Initiation of enteral feeding devices in the intensive care setting

\section{Lee Siew Kum, Goh Annie, Kong Yoke Kuan, Lee Ang Noi}

KK Women's and Children's Hospital, Singapore, Singapore

Introduction: Traditionally, parenteral syringes, tubing and pumps are used for enteral feeding. Using parenteral syringes to administer enteral liquids pose a risk of misadministration intravenously and result in fatal consequences.
In view of high volume of patients requiring enteral feeding via nasogastric tube, the mandatory use of enteral feeding system for pediatric patients is necessary to prevent misadventure.

Objectives: To eliminate risks associated with cross connections of enteral devices with those intended for IV access devices to prevent catastrophic consequences.

Methods: FMEA (Failure Mode Effect Analysis) model is used to identify whether our current enteral feeding practice compromise patient's safety. Sub-processes and potential failure modes, causes and effects are scrutinized. Using the scoring system, it clearly indicated that the delivery process has the greatest potential for hazardous errors on the patients. The organization decided to change the existing enteral feeding devices to designated enteral feeding system.

Results: Mandatory and standardized use of enteral system makes delivery of enteral feeding to patients safer. With this "forcing function' design, errors are avoidable. Post implementation of designated enteral feeding devices yielded significant risk reduction of $83 \%$, which indicate the success of FMEA in minimizing patients' harm.

Conclusions: Use of designated enteral feeding system enhances patient safety. Mortality and morbidity of the patients associated with misconnection will be eliminated; thus potential legal liability and unnecessary length of stay will be reduced.

Reference: Guenter P, Hicks RH, Simmons D, et al. Enteral feeding misconnections: a consortium position statement. Jt Comm J Qual Patient Saf. 2008;34:285-292.

Critical care environment - pedagogy of connectedness

\section{Irene J. Kearns}

University of Johannesburg, Johannesburg, South Africa

Introduction: "More deaths than discharges." Such an outcry is not strange taking into account the situation of the nursing profession, with a diversity of challenges.

Objectives: Pedagogies of connectedness aim to influence motivate and inspire students to learn by connecting theory to praxis. According to Hallowell (2002) connectedness implies certain responsibilities such as not to harm other people or ourselves, to be considerate, caring and accepting and to act within the value system of the nursing profession. Nursing students engage with the reality at the bedside of the patient where learning mostly occurs, away from simulation rooms and laboratories. Furthermore, diversity within the South African context should enrich us - so that we render holistic nursing care, striving to uphold the nurse's pledge.

Methods: The research design is qualitative, explorative and contextual in nature. Data collection is in the form of narratives, from a diverse group of undergraduate students on their most fulfilling and most unfulfilling day in the ward and ICU environment.

Results: Tesch's method of data analysis were followed - by an expert in qualitative data analysis. The primary aim of this article is to communicate the five themes that emerged from the critical care environment:

\section{- $\quad$ structured holistic patient care - positive patient outcomes \\ - active participation \\ - $\quad$ knowledge/skills beyond ICU \\ - knowledge sharing \\ personal growth/fulfillment}

Conclusions: A pedagogy of connectedness is influenced by the meaning and value attached to theory - practice integration and fulfilling clinical life experiences that yield positive patient outcomes. "More discharges then deaths." 
Cost allocation-a model and tool for comparison of nursing care intensity and costs in three ICUs in Norway

\section{Siv K Stafseth $(1,2)$, Lisbeth Fagerstrøm (3)}

(1) Division of Emergencies and Critical care,Dept.of Research and Development, Oslo University hospital, Oslo, Norway; (2) Institute of Clinical Medicine, Faculty of Medicine, University of Oslo, Oslo, Norway; (3) Buskerud University College, Drammen, Norway

Introduction: Allocation of costs in health care and especially the cost of nursing staff in the Intensive Care Units (ICUs) is rising. Attention has been given to the definitions of workload and nursing care intensity. Studies of benchmarking have highlighted the costs in the ICUs often without using nursing scoring systems; Nursing Activities Score is suggested by Reis Miranda \& Jegers(2012).

Objectives: The aims of this study were to identify indicators of resources and to demonstrate nursing care intensity in a model for comparison of ICUs.

Methods: A direct method of cost allocation in ICUs, proportionally to the patients and nursing care intensity cross-sectional within Norway, was used. A model was innovated for describing nursing care intensity and costs by using preexisting hospital data sets. In the method four phases were present: the preparatory, the modeling, the building and the testing phase with implementation.

Results: For each of the components and indicators, an exact definition of item was identified, resulting in uniform reporting templates. Indicators for nursing care intensity and costs were settled. The model was derived for retrospective utilization and resulted in a tool for comparison of ICUs by using Nursing Activities Score.

Conclusions: Cost allocation in ICUs still remain a challenging process. In this study we could demonstrate that it is possible to construct an automatically scripts of data and the model will lower burden for data collection and save time.

References: Reis Miranda D, Jegers M. Monitoring costs in the ICU: a search for a pertinent methodology. Acta Anaesthesiol Scand 2012;56:1104-1113.

\section{Patient mobilization techniques for ICU nurses}

\section{Diana Solms (1), Siv K Stafseth (2)}

(1) Divison of Emergency and Critical Care, Oslo university Hospital, Oslo, Norway; (2) Divison of Research and Development, Divison of Emergency and Critical Care, Oslo university Hospital, Oslo, Norway Introduction: Nurses all over the world have a challenging workload, especially physiological. The patients in the ICU have technical devices, lines and sometimes a heavy bodyweight. After many hours on duty, years and mobilization patients, some will get problems with pain in neck and shoulder. Sick leave can be a big burden and connected to costs for the ICUs. We wanted to mobilize the patients without harm for either, the patient and the nurses.

Objectives: The aim of the project was to reduce the neck and shoulder pain for the nurses, and hopefully reduce number of sick days in the ICU.

Methods: Educational program, to all colleagues, in one ICU at Oslo university hospital, in how to use diffrent techniques for patient mobilization and how to use equipment which can reduce heavy lifting (1). Three trained instructors held theoretical and practical training in 8 hours sessions. Follow-up survey after 1,5 years. Responsrate above $50 \%(n=52)$. Foundings and support was from the Norwegian Labour and Welfare Service (NAV).

Results: Nurses found the program useful and they had less neck and shoulder problems. Several had started to exercises at the gym on private initative. We got a reduce rate in sick leave of $3 \%$.

Conclusions: Nurses have a reduce rate of pain problem after learning patient mobilization techniques and how to use the additional equipment. We will continue this educational program in the future.

Reference: (1). Lunde, P. H., Forflytningskunnskap - Aktivisering, hjelp og trening ved forflytning. Gyldendal Akademiske Norway, 2010

Critical Care Nurses Opinions Regarding Continuous Professional Development, The South African Perspective

\section{S. Ann Young, Shelley Schmollgruber}

University of the Witwatersrand, Parktown, Johannesburg, Gauteng, South Africa

Introduction: Post registration nursing continuing professional development (CPD) provides safe quality patient care by updating critical care nurse's current knowledge and skills. The South African Nursing Council (SANC) has not as yet introduced an accrediting CPD system for post registered nurses.

Objectives: To describe critical care nurses opinions regarding CPD, current extent of their participation in CPD programs and perceived barriers to CPD programs.

Methods: The National Council for the Professional Development of Nursing and Midwifery, Ireland, (2004) questionnaire was adapted for the South African context. The design was a two phase quantitative, non experimental, descriptive survey. A critical care nurse expert panel evaluated the questionnaire in Phase 1. Phase 2, the survey study, represented opinions of critical care nurses working, permanent and/or non-permanent, in level 2 and level 3 Intensive Care Units (ICU) in Gauteng public and/ or private hospitals.

Results: 100 questionnaires were distributed, 71 were returned (response rate $71 \%$ ). Critical care nurses identified the need for knowledge and skills related to new technology $(98.59 \%)$, prefer onsite training with structured training time $(74.65 \%)$, and want recognition from SANC (70.42\%). Access to information of CPD events needs to be communicated to critical care nurses $(83.10 \%)$.

Conclusions: Critical care nurses are internally driven to participate in CPD programs even though there is no accredited SANC program and barriers do exist to participation. Stakeholders, Critical Care Nursing Forum and critical care nurses to develop and support nurses in ICU with a CPD program and to improve patient care.

Lymphocyte expression of apoptotic markers associates with pain ratings in critically ill patients

Elizabeth Papathanassoglou (1), Meropi Mpouzika (2, 4), Margarita Giannakopoulou (2), Evangelos Bozas (2), Sofia Mpoti (3), Nicos Middleton1, Andreas Karabinis $(3,5)$

(1) Cyprus University of Technology, Limassol, Cyprus; (2) University of Athens, Department of Nursing, Athens, Greece; (3) Genimatas General Hospital, Athens, Greece; (4) Technological Educational Institute, Department of Nursing, Athens, Greece; (5) University of Athens, School of Medicine, Athens, Greece

Introduction: Lymphocyte apoptosis in critical illness is associated with immunosuppression. Although pain has been recognized as a pivotal modulator of immunity, in critical illness there is a lack of pertinent evidence.

Objectives: To explore associations between pain ratings and expression of the apoptotic receptor Fas and Fas ligand (FasL) on $\mathrm{B}-$ and T-cells in critically ill patients.

Methods: Thirty-five critically ill patients were followed for 14 days. Lymphocyte Fas/FasL expression in patients were compared to 35 healthy matched controls. Pain was assessed by the self-reported numeric rating scale (NRS), nurse-investigator's NRS assessments, and the behavioral pain scales developed by Puntillo et al (1997) and Payen et al (2001). Severity was assessed by the MODS scale. Lymphocyte Fas/FasL expression was assessed by flow-cytometry. 
Results: Fas and FasL expression on T-helper, T-cytotoxic cells and Fas expression on B-cells were higher $(p<0.0001-0.03)$ in critically ill patients, indicating susceptibility to apoptosis. On the first day, significant associations between Fas expression on B-cells and pain ratings on the nurse-reported NRS ( $r=.530, p=0.029)$, Puntillo ( $r=.672$, $p=0.003)$, and Payen $(r=.472, p=0.046)$ scales were noted. These associations intensified during the day of maximum MODS severity. On the day of minimum MODS severity, significant associations were noted between Fas expression on cytotoxic $T$ cells and pain ratings on the nurse-reported NRS ( $r=.441, p=0.04)$, Puntillo $(r=.466$, $p=0.029)$, and Payen $(r=.398, p=0.052)$ scales.

Conclusions: These results suggest a role for pain in lymphocyte survival and activation in critical illness. Several important nursing implications are discussed.

Pilot exploration of the association between lymphocyte counts, apoptotic markers and anxiety symptoms in critical care nurses

\section{Elizabeth Papathanassoglou (1), Maria Karanikola (1), Meropi Mpouzika $(1,2)$, Sofia Mpoti $(3)$}

1Cyprus University of Technology, Department of Nursing, Limassol, Cyprus, 2Technological Educational Institute, Department of Nursing, Athens, Greece, 3Genimatas General Hospital, Athens, Greece

Introduction: Research evidence suggests an association between stress and anxiety and apoptosis in lymphocyte subsets.

Objectives: To explore potential associations between anxiety symptoms and total and subset lymphocyte counts as well as lymphocyte expression of Fas and Fas ligand (FasL) in a sample of critical care nurses.

Methods: Descriptive correlational single measurement design with a convenience sample of 39 critical care nurses, employed in an adult ICU in Greece. The Hamilton's Anxiety scale was applied for quantitative assessment of anxiety symptoms. Lymphocyte counts and Fas/FasL expression were assessed by flow-cytometry.

Results: The degree of anxious mood $(2.13 \pm 1.3)$, depressive mood $(2.00 \pm 1.3)$ and sleep disturbances $(2.51 \pm 1.37)$ was mild to moderate. About $50 \%$ of participants manifested severe/ very severe sleep disturbance, whilst $45 \%$, approximately, reported severe or very severe depressive mood. B cell counts correlated inversely with anxious mood $(r=-0.319, p=0.045)$, phobias $(r=-0.312, p=0.04)$, depressive mood $(r=-0.578, p<0.0001)$, and gastrointestinal symptoms ( $r=-0.364, p=0.02$ ). Total lymphocyte counts exhibited a moderate positive association with depressive mood $(r=0.357$, $p=0.032)$, as did $T$ cell counts $(r=0.370, p=0.026)$, and $T$ cytotoxic cell counts $(r=0.496, p=0.2)$. No significant associations were noted with lymphocyte Fas and FasL expression, despite non statistically significant trends for associations between Fas-positive B cell counts and the majority of anxiety symptom scores (anxious mood, tension, phobias, cognitive disturbances).

Conclusions: Despite methodological limitations, these results suggest a role for anxiety in alterations of immunity in critical care nurses and merit further investigation.

Pain intensity and apoptosis markers in intubated critical care individuals with and without apparent tissue injury: a comparative pilot study

Elizabeth Papathanassoglou (1), Eleni Kletsiou (3, 4), Margarita Giannakopoulou (2), Evangelos Bozas (1), M Anastasiou-Nana $(3,4)$, EK Iliodromitis $(3,4)$

(1) Cyprus University of Thechnology, Department of Nursing, Limassol, Greece; (2) University of Athens, Department of Nursing, Athens, Greece; (3) University of Athens, Scholl of Medicine, Athens, Greece; (4) Attikon University Hospital, Athens, Greece
Introduction: The investigation of objective indicators of pain in critically ill adults unable to communicate, is of great interest to intensive nursing. Unrelieved pain enhances apoptosis and apoptotic markers are released in circulation.

Objectives: The aim of this pilot study was to investigate plasma soluble apoptotic molecules levels in critically ill adults and potential associations with perception of pain.

Methods: Prospective correlational study with repeated measurements and cross-sectional comparisons in a convenience sample of intubated critically ill adults with $(\mathrm{n} 1=40)$ and without apparent tissue injury (n2=40). Plasma levels of Fas/FasL were quantified by enzyme-linked immunosorbent assay (ELISA) at intermittent sedation (at the time of the sedation break, 15, 30 and 60 minutes later). The Payen and Puntillo pain scales were used for pain intensity assessment.

Results: Following sedation break increases in pain intensity scores were observed in both groups. In patients with apparent tissue injury a significant increase in Fas levels was observed $30 \mathrm{~min}$ after the sedation break (M1=1132,09 $\pm 522,32 \mathrm{pg} / \mathrm{mlvsM2}=1327,73 \pm 551,27 \mathrm{pg} /$ $\mathrm{ml} \mathrm{p}=0,013)$, and this increase was significantly different between the two groups (M1 $=1327,73 \pm 551,27 \mathrm{pg} / \mathrm{ml}$ vs $\mathrm{M} 2=957,90 \pm 539,92 \mathrm{pg} / \mathrm{ml}$ $p=0,031$ ). Difference between groups was also observed in Fas levels at the 15' $(M 1=1294,27 \pm 518,34 \mathrm{pg} / \mathrm{ml}$ Vs $M 2=988,58 \pm 521,42 \mathrm{pg} /$ $\mathrm{ml} \mathrm{p}=0,050$ ) measurements. Pain intensity correlated with plasma levels of Fas $(r=0,385, p=0,001)$ and FasL $(r=0,268, p=0,011)$ in both groups. These associations were controlled for disease severity.

Conclusions: This is the first report of use of plasma levels of Fas and FasL as a pain intensity biochemical markers appropriate for objective pain assessment in uncommunicative critically ill adults.

Serum stress neuropeptide levels associate with lymphocyte expression of apoptotic markers in critical illness

Elizabeth Papathanassoglou (1), Meropi Mpouzika (2, 3), Margarita Giannakopoulou (2), Evangelos Bozas (2), Sofia Mpoti (4), Nicos Middleton (1), Andreas Karabinis $(4,5)$

(1) Cyprus University of Technology, Department of Nursing, Limassol, Cyprus; (2) University of Athens, Department of Nursing, Athens, Greece; (3) Technological Educational Institute, Department of Nursing, Athens, Greece; (4) Gennimatas General Hospital, Athens, Greece; (5) University of Athens, School of Medicine, Athens, Greece

Introduction: In critical illness, apoptotic loss of immunocytes is associated with immunosuppression. Stress-neuropeptides are pivotal modulators of immunity and apoptosis.

Objectives: To explore: A) expression of the apoptotic receptor Fas and Fas ligand (FasL) on B- and T-cells from critically ill patients and associations with disease severity. B) associations between neuropeptide Y (NPY), cortisol, ACTH and prolactin and Fas/ FasL expression.

Methods: Thirty-five critically ill patients (14-day follow-up) were compared to 35 matched controls. Disease severity was assessed (MODS). Immunophenotyping of peripheral blood mononuclear cells was performed by flow-cytometry. ACTH, prolactin serum levels were quantified by an Electrochemiluminescence Immunoassay (ECLIA) and NPY levels by an enzyme-linked immunosorbent assay (ELISA). Fas/ FasL expression in lymphocytes was standardized for viable cell counts.

Results: Fas/ FasL expression on T-helper ( $p:<0.0001-0.03$ ), T-cytotoxic $(p:<0.0001-0.002)$ and Fas expression on B-cells $(p:<0.0001-0.03)$ were higher in critically ill patients. MODS scores associated with FasL expression on cytotoxic T-cells $(r=0.752-0.902$, $p=0.023-0.037$ ). Regarding first day measurements, an inverse association between NPY levels and Fas expression on helper- 
T-cells ( $r=-0.447, p=0.019)$ was noted. On the day of maximum severity, NPY levels associated with FasL expression on helper $(r=-$ 0.733, $p=0.016)$ and cytotoxic $(r=-0.862, p=0.003)$ T-cells. Cortisol levels associated with FasL expression on helper-T-cells $(r=0.804$, $p=0.001)$, and with counts of FasL-positive helper $(r=0.828, p<0.001)$ and cytotoxic $(r=0.544, p=0.04)$ T-cells.

Conclusions: These findings may have significant implications for critical care nursing. Relevant interventions may be needed to support physiologically relevant levels of NPY and to normalize stress-neuropeptide levels in critical illness.

\section{Development of a preterm infant kangaroo care protocol}

Eunjung Lee (1), Eunsook Kim (1), Yu-nah Lee (1), So Hyun Park (1), Geummoon Lee (1), Soonja Oh (1), Jiyoon Lee (1), Young Hee Yi (2)

(1) Samsung Medical Center NICU, Seoul, Republic of Korea; (2) Department of Clinical Nursing Science, Samsung Medical Center, Sungkyunkwan University School of Medicine, Seoul, Republic of Korea

Introduction: In Republic of Korea, $42.3 \%$ of Neonatal Intensive Care Unit(NICU) nurses haven't experienced the Kangaroo Care(KC). The Korean-type KC protocol hasn't existed.

Objectives: This study aimed to develop a preterm infant KC protocol that can guide the practice of NICU nurses.

Methods: A literature experts group consisting of six individuals went through 526 studies published since 2000. 73 studies were selected and each of the studies was evaluated for the evidence level and recommendation strength. After analysis, developed protocol. That was sent to seven experts for content validity. To decide the clinical suitability, protocol was applied to 27 preterm infants, during $\mathrm{KC}$, the Heart Rate (HR), Respiration Rate (RR), Oxygen saturation (SpO2), Body Temperature (BT) were searched. Parents' anxiety and 60 nurses' awareness on the KC were investigated beforeafter application of protocol. Protocol was concluded through final modification work.

Results: After application of the protocol(CVI=.96), HR, BT were significantly increased within normal range than before $(p<.00)$. RR $(p=.387)$, SpO2 $(p=.087)$ weren't significant differences. The anxiety degree of parents was decreased significantly $(p<.00)$. The nurses' awareness wasn't significant difference $(p=.452)$. But among the awareness measuring categories, ' a nurse tries to encourage parents to perform the $\mathrm{KC}$ and to help them' was significant increased $(p<.018) .45$ subcategories protocols in five fields as 'onset criteria', 'training', 'preparation', 'infant's transfer to the parents from Incubator/ICS' and 'infant's transfer to Incubator/ICS from the parents' were determined.

Conclusions: 45 protocols that have been developed to be useful, reliable and comprehensive will be a contribution to quality development of the premature infant nursing care.

The effect of an education on attitudes toward, coping with death, perception of and performance in end of life care among critical care nurses

JungYeon Kim (1), MiYeon Pyo (1), SangHee Kim (2), IIHak Lee (3), YounKyoung Kil (1), EunSook Lee (1), HyangSook Kim (1)

(1) Severance Hospital, Yonsei University Health System, Seoul, Republic of Korea; (2) College of Nursing, Yonsei University, Seoul, Republic of Korea; (3) College of Medicine, Yonsei University, Seoul, Republic of Korea

Introduction: As number of death in hospital increases, endof-life (EOL) care becomes more important. However, evidence demonstrates that nurses are unprepared to deal with it (1).

Objectives: This study evaluated how educational session could affect the attitude toward death and coping, perception and performance EOL care.

Methods: This study used a two-group, pretest-posttest, quasiexperimental design. The participants in education program were 34 critical care nurses from a voluntary group from 1 university-affiliated hospital. The 127 control samples were recruited from others. 4 sessions of educational intervention modified End-of-Life NursingEducation-Consortium (ELNEC)-Critical and the Improving Palliative Care in the ICU (IPAL-ICU).

Results: There was a significant difference $(p<0.05)$ between posttest scores on perception of EOL care. The score of the experimental group (170.03) who received the educational intervention was significantly higher in perception of EOL care than that of those (164.79) who did not receive the education. But there wasn't a significant difference between post-test scores on attitude toward death, coping with death, and performance regarding EOL care.

Conclusions: Because the education could change only the perception regarding EOL care, we need to establish the administrative strategies focusing on changing attitudes, improving coping, and performance regarding EOL care (2).

References: (1). Jeon MH. Recognition among Nurses and Doctors about the Death in ER \& ICU[Dissertation]. Seoul: Hanyang Univ.; 2008. Korean; (2). Noh SH. A study on death perception, terminal care attitude, and terminal care performance of clinical nurses caring for cancer patients[Dissertation]. Seoul: Sungkyunkwan univ.; 2010. Korean.

Participant perceptions of the Nepean ICU multidisciplinary team meeting

\section{Hailey Samuels, Leonie Weisbrodt}

Nepean Hospital, Penrith, Australia

Introduction: The functioning of multi-disciplinary teams (MDT) in the complex, high stress environment of a busy intensive care unit relies on good inter-professional collaboration, effective communication and an appreciation and respect of the expertise each specialty brings. Ineffective communication can lead to adverse events, delays in treatments and poorer outcomes for patients. To promote good communication, weekly MDT meetings have been introduced into our Intensive Care Unit. Patients who have been admitted for more than twenty-one days are reviewed and long term treatment plans are developed. The team includes a senior medical officer, bedside nurse, allied health care team and occasionally a member of the patient's family.

Objectives: In an effort to streamline care delivery and understand barriers within the ICU multidisciplinary team a survey was conducted to gauge team member perception of the function and effectiveness of the weekly meetings.

Methods: The survey was a developed via Survey monkey using likert-style level of agreement statements. $100 \%$ of MDT members responded to the survey $(n=6)$.

Results: Overall there was agreement that the multidisciplinary team meetings function well. It was felt by all participants that the attendance of a senior medical officer was paramount to allow on the spot decisions to be made. Most participants indicated that attendance should be prioritised but barriers, such as overall workload, time constraints and staff shortages were barriers to attendance. There was some uncertainty about the improvement in patient outcomes since the introduction of the multidisciplinary meetings as further research is required in this area. 


\section{Understanding decisional conflict in organ donation}

\section{Maryn Reyneke, Ronel Pretorius, Petra Bester \\ North-West University, Potchefstroom, South Africa}

Introduction: Despite the achievement of and media coverage around the first heart transplant conducted in South Africa, deceased organ donation remains critically low. The complexity of organ donation seems to be influenced by amongst others, the universal process of choosing between alternative courses of action or inaction, also known as decisional conflict. To that, ambivalent decision-making styles of relatives are considered a risk factor for traumatic memories.

Objectives: To explore the decisional conflict experienced by relatives having to decide on organ donation. Methods: We interviewed eight relatives exposed to the living experience of decision-making regarding organ donation through in-depth individual interviews by means of the interpretive description method.

Results: The results identified seven shared themes that described the relatives' decisional conflict while being confronted with organ donation. Among these themes is the impact of sudden death and dying, the cause of death, the diagnosis and the unnatural reality of brain death. Relatives found themselves in a psychological abyss with conflicting thoughts of finality, fear, hope and regret and cope by projecting information, beliefs and thoughts against various reasoning.

Conclusions: The shared realities of the relatives and highlighted themes provide insight and understanding of their experiences during the decision making process of organ donation. Emotional support given by critical care staff and transplant coordinators based on this insight might ease the decision-making process for relatives. This could well increase organ donation through family consent.

Photographic storyboards: preparing a mother and child for cardiac surgery

\section{Angela Leonard, Minette Coetzee}

University of Cape Town, Cape Town, South Africa

Introduction: In South Africa, a multicultural, multilingual, low health literacy society, a mother may be excluded from accessing health information relevant to her child's hospitalisation. Being adequately prepared and in possession of sufficient knowledge is known to relieve anxiety and may assist a mother in best preparing her child for any procedures or surgeries.

Objectives: The process of cardiac surgery is complex but a relatively predictable pathway; assessment, to admission, theatre, PICU and back to the ward. A need was identified for succinct, accessible information about both the cardiac procedure and pathway.

Methods: The methodology was intentionally participative and included working with parents, children, cardiac surgeons and nurses. Four options of information delivery were presented to mothers; a storyboard of photographs, a doll simulating a baby postoperatively in PICU, a medical information sheet and an anatomical picture of a heart.

Results: Rapid appraisal questionnaires and focus groups with both pre and post-operative mothers led to the storyboard being chosen. It consisted of twelve photographs documenting a 6 year old child's journey from admission for cardiac surgery until discharge.

Conclusions: Specific evaluation of the storyboard highlighted that parents felt empowered by it, giving them a sense of hope and awareness of what to expect. Knowledge of and familiarity with equipment provided parents with greater understanding and thus alleviated their stress. The photo storyboard was identified as being assessable and preferable in preparing parents and children for cardiac surgery. This poster will present the processes of development and evaluation of the photographic storyboards.
Development and preliminary validation of a new questionnaire to evaluate factors influencing nurses' decision-making (FINDEM) in critical care

Elizabeth Papathanassoglou (1), Vassiliki Karra (2 ,3), Chrysoula Lemonidou (2), Margarita Giannakopoulou (2)

(1) Cyprus University of Technology, Department of Nursing, Limassol, Cyprus; (2) University of Athens, Department of Nursing, Athens, Greece; (3) Sismanogleion Hospital, Intensive Care Unit, Athens, Greece

Introduction: The Factors Influencing Nurses' Decision-Making (FINDEM) questionnaire was developed for assessing the importance that critical care nurses assign to factors contributing to their clinical decision-making.

Objectives: To assess validity and reliability of FINDEM questionnaire.

Methods: The FINDEM questionnaire resulted from focus groups discussions. It was pilot tested in 2013 to a convenience sample of 196 critical care nurses in Greece and consists of 60 items which are expressed on a 5-point likert scale. Principal component analysis with Varimax rotation was conducted to assess the factor structure of the questionnaire. Validity was further examined by investigating the intercorrelations of the subscales. Assessment of internal consistency reliability and test-retest reliability followed.

Results: Factor analysis indicated an 8-dimensional model that explained $51.8 \%$ of the total variance: Task complexity \& Task conditions, Knowledge \& Knowledge Awareness, Accountability, Attributes of ICU management, Knowing the Patient, Attractive ICU Work Environment, Patient's Characteristics and Clear Professional Responsibilities. All subscales exceeded the minimum reliability standard of 0.70 . Almost all intercorrelations were significant with correlation coefficients ranging from 0.23 to 0.53 . "Task complexity and Task conditions" subscale scored higher (mean: 4.4 \pm 0.4 ) compared to the correspondence mean scores of other subscales indicating that critical care nurses assign the greatest importance on understanding the clinical problem and the decision-making conditions. Accountability scored the lowest (mean: 2.1 \pm 0.6 ) indicating that it potentially has the less important role in nurses' decision-making.

Conclusions: The FINDEM questionnaire is a reliable and valid instrument to evaluate factors related to critical care nurses' decisionmaking.

Nursing workload in the intensive care unit: a multicentre study using the Nursing Activities Score (NAS)

Katia Padilha (1), Siv Stafseth (2), Marga Hoogendoom (3), Javier Carmona (4), Konstantinos Giakoumidakis (5), Om Rageb (6), Edyta Cudak (7), Regina Sousa (8), Ricardo Barbosa (9), Dinis Reis Miranda (10), Cristiane Santoro (11)

(1) School of Nursing-University of São Paulo, São Paulo, Brazil; (2) Oslo University Hospital, Oslo, Norway; (3) Department Anesthesiology \& Intensive Care - Isala Klinieken, Zwolle, The Netherlands; (4) Hospital Universitário Fundación Alcorcon, Madrid, Spain; (5) Evangelismos General Hospital of Athens, Athens, Greece; (6) Faculty of Nursing-Sohag University, Assuan, Egypt; (7) University of Medical Sciences, Poznan, Poland; (8) School of Nursing-University of São Paulo, São Paulo, Brazil; (9) School of Nursing-University of São Paulo, São Paulo, Brazil; (10) Health Care Profissional \& Independ Hospital, Bruges, Belgium; (11) Osvaldo Cruz Hospital, São Paulo, Brazil

Introduction: High costs of intensive care as well as quality of care and patient safety demand measurement of nursing workload in order to determine nursing staff requirements in Intensive Care Units (ICU). 
Objectives: To describe nursing workload in the ICU from different countries using the Nursing Activities Score (NAS); to analyze NAS and patients variables i.e. age, type of treatment in the ICU, Simplified Acute Physiology Score II (SAPS II) and discharge from the ICU.

Methods: Cross-sectional observational study in 19 ICUs of seven countries (Norway, The Netherlands, Spain, Poland, Egypt, Greece and Brazil) with a total of 776 adult critically ill patients, in November, 2012. The association of NAS and the variables was analyzed by ANOVA, and Chi-Square. Statistical significance was $5 \%$.

Results: Patients from Egypt were younger (40.2 years, $p<0.000)$ and presented a higher mortality in the ICU $(33.3 \%, p<0.000)$ while in Poland patients were more severe (SAPSII $=65.29$ points, $p<0.000$ ) comparatively with patients from other countries. The surgical treatment was predominant in all countries, except in Brazil. The total NAS mean were $65.3 \%$ varied from $46.9 \%$ (Spain) to $101.7 \%$ (Norway). There was a similar NAS in the following countries: Spain and The Netherlands (46.9\% e $51.5 \%$ ), Egypt and Greece $(57.0 \%$ and $60.1 \%)$, Brazil and Poland $(68.1 \%$ and $70.1 \%)$. Norway $(101.7 \%)$ had the highest nursing workload but lowest mortality $(2.4 \%)$.

Conclusions: This study shows a high nursing workload in the ICUs. So, improvement in a nursing staffing is required to ensure safety and quality in ICU.

Reference: Miranda DR, Nap R, Rijk A, Schaufeli W, lapichino G. Nursing Activities Score (NAS). Crit Care Med. 2003;31(2):374-82.

Pathways to care of the critically ill child - a nursing perspective

Rencia Gillespie (1), Peter Hodkinson (1), Alison Ward (2), Lee Wallis (1), Brenda Morrow (1), Andrew Argent (1)

(1) University of Cape Town, Cape Town, South Africa; (2) Oxford University, Oxford, UK

Introduction: Data on critically ill children admitted to the PICU at the Red Cross War Memorial Children's Hospital in Cape Town suggests that there may be problems during the "pathway" to care, from first contact with medical services through to PICU admission.

Objectives: To identify pathways to care of critically ill or injured children, and link this with the role of nurses.

Methods: Data on 280 children admitted for emergency reasons to the PICU of Red Cross War Memorial Children's Hospital (RCWMCH) and those who died in RCWMCH emergency unit and nearby facilities were prospectively collected over a one-year period.

Results: Nearly all children were seen at various healthcare facilities. These included clinics, community health centres, district, regional and tertiary referral hospitals. Nursing staff were involved at every step of the pathways, except the emergency transport of patients between institutions. Nurses were primarily responsible for triage and in many cases for initial clinical assessment, administration of therapy, assistance with resuscitation and ongoing monitoring of vital signs. Nurses were also responsible for communication with emergency medical services and ongoing observation of the children while awaiting transportation. Various nurse categories were involved, however their paediatric emergency care training and experience is unknown.

Conclusions: When considering the role of the nurse in the care of the critically ill child, it is clear that nurses have a wide variety of roles in very different

Factors affecting cardiopulmonary and critical care practice among physiuotherapists in South West Nigeria

Nse Odunaiya (1 ,2), Kemi Adeniran (1), Wale Badru (1)

(1) University of Ibadan, Ibadan, Oyo state, Nigeria; (2) Stellenbosch University, Cape town, South Africa
Introduction: Critical care involves team work for quality delivery of care and cardiopulmonary Physiotherapists play pivotal role in critical care. Respiratory therapy has been considered the primary role of physiotherapist in critical care. The extent of practice of cardiopulmonary and critical care has been reported to be low among Physiotherapist in South West Nigeria and proficiency of Nigerian trained Physiotherapists in cardiopulmonary techniques and skills needed in critical care is lacking.

Objectives: This study was conducted to determine factors affecting cardiopulmonary and critical care practice among physiotherapist in Nigeria.

Methods: The study was a cross sectional study and participants were 65 physiotherapist practicing in selected teaching hospitals in south west Nigeria. Ethical approval was obtained and participants informed consent were obtained. An instrument designed by the authors and validated for content by a panel of experts was used to collect data. Qualitative component exploring some of the factors responsible for low level of cardiopulmonary and critical care practice is ongoing and will be reported at the conference

Results: Factors causing low level of practice include lack of referral for physiotherapy by doctors, lack of facilities for critical care and lack of proficiency.

Conclusions: Nigerian trained Physiotherapists involvement in Critical care is low due to lack of referral from doctors, lack of facilities for critical care in selected Hospitals and lack of interest in critical by Physiotherapists low level in south west Nigeria among Physiotherapists. Recommendation: team work, training, motivation quality assurance are strongly recommended in Nigeria.

Family presence during daily inter-professional rounds

\section{Nicky Holmes, Barb Duncan, Anita Au}

Sunnybrook Health Sciences Centre, Toronto, Ontario, Canada

Introduction: "Family-centered care incorporates a partnership between families and health care providers when caring for the patient" (Cypress, 2012).

Methods: In keeping with this philosophy the critical care units at a regional trauma centre in Toronto, Canada introduced the presence and participation of family during daily inter professional rounds. Although implementing this change in practice was somewhat challenging it has become an integral part of facilitating communication between families and the health care team. Cypress (2012) defines family presence on rounds as "family members in the patient's room during a discussion of the patient's medical condition by the health care team........ The patient and the family are engaged and family members participate in the discussion". In 2004 the American College of Critical Care Medicine made recommendations encouraging the presence of family on rounds. Despite this only one research study in a medical critical care unit has been conducted (Cypress, 2012).

Results: While we have not conducted any studies exploring the impact of actively involving families on rounds, anecdotally we have observed what an effective tool it is in building trust, enhancing communication and patient/family satisfaction. Thereby decreasing anxiety in families who are in crisis because they are dealing with a critically ill loved one.

Conclusions: This presentation will focus on the benefits of introducing the family centered care model of family presence on rounds. We will discuss the opportunities for further research as well as our experiences and common misconceptions encountered when changing a unit culture to embrace this standard of care. 
Addressing management challenges in a large intensive care unit

\section{Mokgadi Matlakala \\ UNISA, Pretoria, South Africa}

Introduction: There seems to be no strategies available to assist nurses to manage large ICUs, and unit managers deal with problems as they arise.

Objectives: To illustrate the need for and provide the strategies that will enhance sustainable management of a large ICU.

Methods: A qualitative, exploratory and descriptive research was conducted. Data were collected through individual interviews from a purposive sample of unit managers from ICUs of five hospitals in Tshwane metropolitan, Gauteng Province; and analyzed using thematic coding.

Results: The challenges reported for compilation of evidence in preparation for development of the strategies were related to unit sizes and layout, shortage of trained and experienced intensive care nurses, shortage of equipment and supplies, and the stressors in the unit. The strategies were developed for the challenges identified.

Conclusions: ICUs need measures to drive the desired strategies into actions to improve the management of the unit continuously. Future research should be aimed at investigating the effectiveness of the identified strategies.

The relationship between perceived physical health and adherence to heamodialysis among end stage renal disease patients at a local heamodialysis center in Harare, Zimbabwe

Geldine Chironda (1), Rudo Nyamakura (2), Busisiwe Bhengu (3) (1) University of Kwazulu Natal, School of nursing and public health, Durban, South Africa; (2) University of Zimbabwe, College of Health Sciences, Harare, Zimbabwe; (3) University of Kwazulu Natal, School of nursing and public health, Durban, South Africa

Introduction: Problems of adherence to haemodialysis among End Stage Renal Disease (ESRD) patients have been observed at a local haemodialysis centre in Harare, Zimbabwe. Poor adherence to haemodialysis is also apparent in regional and international literature. Perceived physical health appeared to be the likely contributing factor to poor adherence to haemodialysis.

Objectives: This study aimed at describing and examining the relationship between perceived physical health and level of adherence to haemodialysis among ESRD patients. The Activities of Living Model guided this study.

Methods: A descriptive correlational study design was used. A simple random sample of 85 participants was selected. Data was collected using three instuments developed by the investigator in the form of interview schedule namely the Demographic Data, The Adherence to Haemodialysis and the Perceived Physical Health. Descriptive statistics, the Pearson's correlation coefficient and linear regression were used to analyse the data.

Results: Eighty-three (97.6\%) demonstrated low adherence to haemodialysis. Eighty - two $(96.5 \%)$ perceived their physical health to be average. The Pearson correlation coefficient $(r=.44 p<.01)$ showed a weak negative significant relationship. Perceived physical health had an impact of $19.7 \%$ on adherence to haemodialysis (R2 0.197).

Conclusions: The findings predict that as perceived physical health increase, adherence to haemodialysis decrease among ESRD patients. Recommendation: Medical surgical nurses should intensify individualized client programmes to correct ESRD clients' perceptions and identify other contributing factors to poor haemodialysis.
Barriers to the implementation of evidence-based practices in a critical care unit

\section{Candice Bowers, Portia Jordan}

Nelson Mandela Metropolitan University, Port Elizabeth, South Africa Introduction: Implementing evidence-based practices in the critical care unit may prove to be an intricate process with many facets hindering success or sustainability. Barriers to implementing evidence-based practices may exist on diverse levels: the characteristics of evidence, the individual or the organization.

Objectives: The study aimed to explore the barriers to the implementation of evidence-based practices in a critical care unit

Methods: A quantitative survey was used to collect data from 70 professional nurses in the private critical care unit.

Results: Only $57 \%$ of nurses searched for evidence occasionally to support clinical decisions linked to nursing the critically ill patient, while $43 \%$ of nurse indicated they would ask their peers/ colleagues, or rely on previous training or intuition when they need information to guide clinical decision-making. Barriers to implementing evidencebased practices were: resistance to change by the nurses (49\%); insufficient time to implement evidence-based practices $(34 \%)$; limited electronic access in the critical care unit $(49 \%)$; too much literature $(40 \%)$ to inform decision making; no dedicated champion to drive evidence-based practice $(34 \%)$ in the critical care units; high patient acuity levels in the critical care unit $(34 \%)$; increased workload in the critical care unit (49\%). The organisational context definitely appears to pose barriers to implementing evidence-base practices in the critical care unit.

Conclusions: The study findings reaffirmed various barriers to the implementation of evidence-based practices in the critical care unit which may compromise the quality of patient care.

The profile of recovery room nurses in Johannesburg hospitals Juan Scribante, Helen Perrie

University of the Witwatersrand, Johannesburg, South Africa

Introduction: Apart from anecdotal evidence, little is known of the recovery room nurses (RRN) in South Africa.

Methods: An exploratory, prospective descriptive pilot study was done in the recovery rooms of six Johannesburg hospitals: three academic and three private hospitals. Data were collected from senior nursing personnel.

Results: Nurse: patient ratios were difficult to determine. Agency staff was used by one recovery room. The other recovery rooms used their permanent staff to work overtime. All the hospitals used anaesthetic nurses to double up when necessary. Only one of the recovery rooms had a supernumerary anaesthetist available. A total of 49 nurses were working in the recovery rooms. The majority, $95.9 \%$ $(n=47)$ of the RRN were females. The average ages of the RRN were 44 years (25-63 years), median 41 years. The experience of the RRN ranged from 1 month to 35 years with an average of 8.6 years. The majority of nurses, $57.1 \%(n=28)$ were professional nurses, and $42.9 \%(n=21)$ were enrolled nurses. Of the 28 professional nurses $32 \%(n=9)$ had no post graduate training. The remaining 19 nurses had the following post graduate qualifications: management and operating room technique $17.9 \%(n=5)$ respectively, critical care $14.3 \%(n=4)$ and education $10.7 \%(n=3)$. The six month anaesthetic nurse qualification that is not an official South African Nursing Council qualification, was held by $35.7 \%(n=10)$ of the professional nurses and by $4,8 \%(n=1)$ of the enrolled nurses.

Conclusions: There is a need to establish a national profile of RRN in South Africa. 
Implementation of antimicrobial copper in a neonatal intensive care unit (NICU)

Marina Anagnostakou (1), Evaggelia Kouskouni (3), Chrysa Petropoulou (1), Panos Efstathiou (2), Katerina Karageorgou (2), Zacharoula Manolidou (2), Spiros Papanikolaou (2), Elias Logothetis (3), Vassiliki Karyoti (2)

(1) "Agia Sophia" Children's Hospital (NICU), Athens, Greece; (2) National Health Operations Centre Ministry of Health, Athens, Greece; (3) Medical School of the University of Athens, Microbiology laboratory of Aretaieio Hospital, Athens, Greece

Objectives: The aim of this study was to investigate the effectiveness of the application of antimicrobial copper alloys $(\mathrm{Cu}+)$ in a Neonatal Intensive Care Unit (NICU) in relation to the reduction of microbial flora.

Methods: At a Level III Neonatal Intensive Care Unit of a pediatric hospital, with the capacity of twenty-six (26) incubators, antimicrobial copper $(\mathrm{Cu}+)$ was implemented on touch surfaces and objects. The copper alloy contains Cu 63\% - Zn 37\% (Lead Low). Microbiological cultures were taken in three different time periods, before and after the application of $\mathrm{Cu}+$, using dry and wet method technique.

Results: In the above NICU, the reduction of microbial flora after the implementation of the antimicrobial copper $(\mathrm{Cu}+)$ on the selected surfaces and objects was statistically significant $(n=15, p<0,05)$ and was recorded at $90 \%$. The pathogens isolated at high rates (CFU / ml) prior to copper implementation were as follows: Klebsiella spp., Staph. Epidermidis, Staph. Aureus, Enterococcus spp.

Conclusions: This study highlights the positive impact of antimicrobial copper $(\mathrm{Cu}+)$ and demonstrates that copper implemented surfaces and objects are effective in neutralizing bacteria, which are responsible for Health Care Acquired Infections in the nosocomial environment (HCAls). The innovative implementation of antimicrobial copper in the NICU and the significant reduction of microbial flora heralds the reduction of antimicrobial drugs use, and a possible reduction of hospital acquired infections and hospitalization time.

Improving mentoring of third year nursing students in the critical care unit

\section{Renee Kotze, Maria Phillips}

University of the Free State, Bloemfontein, South Africa

Objectives: The purpose of this study was to improve the mentoring programme of the 3rd year nursing students by the students in the postgraduate critical care programme.

Methods: A qualitative consensus research design was chosen and data collection was done through Multiple Nominal Groups. The unit of analysis consisted of 16 third year nursing students. Ethical considerations were strictly adhered to and participants were respected mainly to protect them. Two nominal groups were conducted with eight participants each. Trustworthiness of data was enhanced by a data trial, expert group facilitator and a cocoder. During the qualitative analysis themes were identified and prioritisation of themes was done through a quantitative ranking process according to the steps as described by Van Breda (2005).

Results: The first five themes were clustered under Coordination of Activities and were: Opportunity to Establish a Relationship (21), Mentor and Higher Education Institute (20.5), Control and Organize (16.5), Higher Education Institute (HEI) and Critical Care Unit (13.5) and Mentee and Mentor (9.5). The next ranked theme namely Characteristic of a Mentor (12) focused on the attributes of the mentor as seen from the mentees' viewpoint. The last two themes Skills Development (10.5) and Outcomes (4.5) were clustered together under the Process of Mentoring.

Conclusions: A well organised mentoring program is mainly dependent on the good coordination between HEI, Mentor and Mentee and the CCU in a structured way as demonstrated by the Model of the Mentoring Model. The mentor should allow mentees to develop skills and complete outcomes with a holistic approach.

\section{Understanding decisional conflict in organ donation}

\section{Maryn Reyneke (1), Ronel Pretorius (2), Petra Bester (2)}

(1) Christiaan Barnard Memorial Hospital, Cape Town, South Africa; (2) North West University, Potchefstroom, South Africa

Introduction: Family consent directly influences organ donation rates. The request of organ donation confronts a person with the reality of death and dying. If a persons wishes are not know it makes the decision difficult. A transplant coordinator deals with a family directly and can have an impact on the decision made. The transplant coordinator in South Africa is exposed to a wide variety of cultures and religions, with different perspectives on life, death and organ donation.

Methods: Interpretive descriptive research methodology was used to gain insight into the ways family members of brain death patients described and made meaning of the conflict they experienced. Data collection consisted in-depth interviews and participants interviewed represented the multi-racial population of the Western Cape. With the understanding that human experiences are socially constructed and subjective, the possibility of shared realities among the different families was explored.

Results: The results identified seven shared themes. Among these themes is the impact of sudden death and dying, the cause of death, the diagnosis and the unnatural reality of brain death. Families found themselves in a psychological abyss with conflicting thought of finality, fear, hope and regret and cope by projecting information, beliefs and thoughts against various reasoning.

Conclusions: Family background, culture and gender influence the view point and role of the decision maker, contributing to the understanding of reactions and projections seen during the process. Appropriate emotional support given, might ease the decisionmaking process for family members. This could well increase organ donation through family consent.

Does high-fidelity simulation work? What is the evidence?

\section{Maria Phillips}

University of the Free State, Bloemfontein, South Africa

Introduction: The presentation will describe the features and directions in high fidelity simulation research. Research in simulation is in its infancy and more quantitative studies are required to establish this learning pedagogy with best evidence. The First Research Consensus Summit for simulation set a research agenda and key areas in 2011. Lead researchers in simulation identified topics for research and possible research questions. The use of instruments for simulation research is complex as the proof of validity and reliability are still contentious.

Methods: The methodology and findings of a pilot study to explore this area of research will be presented as an example for future research. We examined the use of instruments to collect data regarding the simulation experiences of 3rd year Baccalaureus nursing students. Their satisfaction and self-confidence after a simulation were measured by means of validated and reliable instruments as recommended by the First Consensus Summit of 2011. Several research instruments are available; however, reliability and validity in some of these must still be established. Findings will be compared to international studies.

Results: Significant findings of international studies will be presented. Conclusions: Most health professions make use of simulation 
as a learning strategy. Trans-disciplinary awareness of research methodologies and findings in simulation will lead to the faster advancement of the science of simulation for the benefit of all.

Reference: Østergaard, D., Dieckmann, P., Issenberg, S. B., Ringsted, C., \& Ostergaard, D. (2011). Setting a research agenda for simulation-based healthcare education: a synthesis of the outcome from an Utstein style meeting. Simulation in healthcare : Journal of the Society for Simulation in Healthcare, 6(3), 155-67. doi:10.1097/ SIH.0b013e3182207c24

Starting a high-fidelity simulation laboratory: pearls and perils

\section{Maria Phillips}

University of the Free State, Bloemfontein, South Africa

Introduction: This presentation aims to inform managers of issues that need to be taken into account when considering setting up a simulation facility.

Methods: Simulation environment design and planning are determined by fiscal means (sources like fundraisings, grants and sponsors), building codes and university policies on architecture and management of equipment. Positions of electric sockets, telephone lines, cameras, microphones, pluming, network cables, security points, bed bells and air cons are negotiated with the various contractors. However these decisions affect the ease of functionality of the facility. The position of equipment, rest rooms, security and the flow of students will furthermore influence the efficiency of the utilisation.

Results: The integration of simulation into programmes requires a vigorous revision of curricula. The human aspect requires identification of the champions, patience, sensitivity regarding peoples comfort levels and building of group cohesion. Novices to simulation facilities may find some of the following issues challenging: streaming, connecting wireless devices, writing and programming scenarios, IP addresses connections, AV devices and troubleshooting skills that only develop over time. Logistics comprise of bookings procedures, software design and programming, logistical planning meetings, template design, storage of footage, and inventory management. The planning of research concerning the effects of simulation for academic and quality purposes are a high priority and confounding variable should be prevented.

Conclusions: The financial cost and human investment establishing a simulation facility mandates effective and quality utilisation of facility.

Reference: Rothgeb MK. (2008). Creating a nursing simulation laboratory: a Literature review. The Journal of nursing education, 47(11), 489-94.

\section{Applications of high-fidelity simulation in critical care}

\section{Maria Phillips}

University of the Free State, Bloemfontein, South Africa

Introduction: Presently, high-fidelity simulation receives intense global and national attention. This results from the concerns about patient safety and quality care. High risk industries like aviation (and many others) use simulation to reduce adverse incidences, to improve skills of communication and valuing each team member's contribution. Likewise, these lessons apply to the critical care team too.

Methods: High-fidelity simulators are wireless full body devices with abilities to present with normal and abnormal conditions with variations in ECG, heart, lung and abdominal sounds, pulse pressure and pupil reactions. Features may also include cyanoses and convulsions, sweating and drug recognition. Skills learners may proof their competence in are; IV/IO catheters and chest tubes insertion, medications administered, intubation, ventilation, CPR, defibrillation and management of traumatic amputations.

Results: During simulations professionals can; clarify their own and one another's roles, practice communication, collaborate in a team and receive constructive feedback. Simulation encounters are video recorded. A safe, confidential and non-judgment environment builds confidence and clinical judgment. Student learning is augmented by debriefing, reflection while watching footage of the experience and peer feedback.

Conclusions: Simulation is effective in the orientation of new staff members, building team competence and collaboration, deliberate practice of skills and management of emergency events in both critical care education and clinical practice.

Reference: Rourke, L, Schmidt, M \& Garga, N 2010, Theory-Based Research of High Fidelity Simulation Use in Nursing Education : A Review of the Literature, Internaltional Journal of Nursing Education Scholarship, vol. 7, no. 1, pp. 1-14.

A model to facilitate constructive patterns of behavior and interaction in critical care units

Suegnet Scholtz, Elzabe Nel, Marie Poggenpoel, Chris Myburgh University of Johannesburg, Johannesburg, South Africa

Introduction: Critical care nurses have adapted to the fast-paced and stressful environment of the critical careunit (CCU), by functioning within their own, unique culture.In order to meet the needs of their patients, these nurses should be whole themselves. Studying this culture provides the key to understandingand the basis for facilitating wholeness in critical care nurses.

Objectives: To explore and describe the culture of critical care nurses and to develop, describe and write guidelines to operationalise and evaluate a model to facilitate constructive patterns of behaviour and interaction in the CCU.

Methods: An explorative approachwith an ethnographic case study design was used. A model was developed in four steps: Concept analysis and identification of the central concept, description ofthe relationship statements, the model and guidelines to implement the model and finally evaluation of the model (Chinn \& Kramer, 2011:235).

Results: The culture of critical care nurses was identified through patterns of behaviour and interaction; Patterns of patient adoption, armour display, despondency because of the demands to adjust, "sibling-like" teamwork and non-support from management and the medical team. The model consists of a guidance, interactive and intervention phase.

Conclusions: Critical care nurses become aware of existing patterns. They develop action plans and implement modified behaviour and interactions. This is followed by a process of analysis, critical reflection and change which leads to constructive patterns of behaviour and interactions in the CCU.

Reference: Chinn, P.L. \& Kramer, M.K. (2011). Integrated theory and knowledge development in nursing.7th edition. St Louis: Mosby Elsevier.

The knowledge, attitude and beliefs of doctors and nurses concerning neonatal pain management

\section{Sizakele Lucia Thembekile Khoza}

Department of Nursing Education, Faculty of Health Sciences. University of the Witwatersrand, Johannesburg, South Africa

Introduction: Effective neonatal pain management is influenced by staff knowledge, attitudes and beliefs.

Objectives: To review current practice in neonatal pain management 
and describe the knowledge, beliefs and attitudes of neonatal staff on pain management in Gauteng hospitals.

Methods: Cross-sectional survey design. Neonatal nurses and doctors at two academic hospitals in Johannesburg completed a modified Infant Pain Questionnaire (Porter et al., 1997; ). Data were collected concerning knowledge of neonatal pain management, attitudes toward pain management in neonates and beliefs about pain management practices.

Results: The response rate of this study was $35.33 \%(n=53)$. The majority of the respondents were professional nurses $(88.68 \%$; $n=47)$ working in neonatal intensive care units $(80.77 \%$; $n=42)$ with $0-5$ $(45.28 \% ; n=24)$ years experience in neonatal care nursing. Absence of pain management guideline in the neonatal units was reported on by $64 \%$ respondents. Statistically significant findings $(p=0.031$, $0.006,0.036,0.006,0.007$ ) were observed on five procedures whereby staff indicated a belief that increased pain severity required increased administration of pharmacological interventions. A review of the pain management interventions utilised in the study setting indicated increased preference for pharmacological interventions to relieve moderate to severe pain.

Conclusions: Despite inconsistencies in the identification and management of neonatal pain, neonatal nurses implemented limited pain interventions within their scope of practice. Recommendations: Optimal pain management requires a deliberate collaborative effort to increase knowledge to impact neonatal practice: neonatal pain management guidelines are the beginning

Open visiting: beliefs and attitudes of intensive care clinicians

\section{Vivien Herbert, Candice Harris, Shelley Schmollgruber}

Department of Nursing Education, Faculty of Health Sciences. University of the Witwatersrand, Johannesburg, South Africa

Introduction: Despite intensive care visitation being research and discussed over the last 25 years, the topic still remains controversial. Open visiting, restrictive visiting and liberalised visiting policies have been the three visiting policies discussed in the relevant literature. In South Africa there has been no studies done that determine the beliefs and attitudes of ICU clinicians (doctors and nurses) regarding visiting policies.

Objectives: The purpose of this study was to determine the beliefs and attitudes of ICU clinicians regarding visiting, visiting policies and open visiting in ICUs with a view to advising changes to existing policies in the best interest of patient care.

Methods: Aquantitative, descriptive, comparative and contextual twophase design consisting of 3 steps was utilised in this study. Phase one (Step 1) involved the validation (Judgement and Quantification Stage of the already developed data collection questionnaire (Beliefs and Attitudes towards Visitation in an ICU questionnaire) BAVIQ using Lynn's model. Six purposively selected ICU clinicians were invited to validate the feasibility of each question and the validity of the entire instrument for use in the South African context. Phase one (Step 2) involved the restructuring of the questionnaire according to the results from the validation of the instrument. Phase two (Step 3 ) as the data collection stage where the restructured questionnaire was handed out to the comparative ICU clinicians for completion. Setting: The setting for this study was the adult ICUs (coronary care, cardiothoracic, trauma, neuro- surgery and multidisciplinary ICU) of a large quaternary public hospital in Johannesburg. The population selected for this study was all ICU doctors and nurses working presently in the ICU setting for at least 4 months. A non- purposive sampling method was used in this study for both phases.

Results: The results of this study showed that there are similarities and significant differences between ICU clinicians' beliefs and attitudes regarding visiting policies. This result could hamper the transformation of a restrictive visiting policy to an open visiting policy in an ICU setting. Due to the existing culture and paternalism of hospital management in these intensive care units there still is resistance to change from the restrictive visiting policy.

Conclusions: The ICU clinicians' main concern for not wanting an open visiting policy in their ICUs was that the safety of the patient and the ICU staff would be in jeopardy especially in Johannesburg were this study was performed. In all the ICUs that were included an open visiting policy is adopted when the patient is dying. Relevance to practice: According to the South African Nursing Council and the American Society of Critical Care Nursing Forum, the vision of nursing care is to provide quality humane nursing care to all patients with the understanding that humane care for the critically ill patient means caring for the patient as a whole person within the context of their family and their community. With having institutions enforcing policies that are non-clinically motivated lacks humanness and is uncaring. 
INDEX

A

Adeniran, Kem

Aitken, Leanne

Akyolcu, Neriman

Anagnostakou, Marina

Anastasiou-Nana, M

Ang, Su Ling

Annie, Goh

Argent, Andrew

$\mathrm{Au}$, Anita

Avsar, Imren

B

Badru, Wale

Barbosa, Ricardo

Bester, Petra

Bhengu, Busisiwe

Bonderup, Anna

Bowers, Candice

Bozas, Evangelos

Bozkurt, Gülçin

Brysiewicz, Petra

Burmeister, Elizabeth

C

Carmona, Javier

Chabeli, Mary

Chan, Yoke Hwee

Chironda, Geldine

Choi, Su Jung

Coetzee, Minette

Cudak, Edyta

D

Davis, Clare

Diacon, Annette

Duncan, Barb

\section{E}

Efstathiou, Panos

Egerod, Ingrid

$\mathrm{F}$

Fagerstrøm, Lisbeth

Fragtrup, Suzanna

G

Giakoumidakis, Konstantinos

Giannakopoulou, Margarita

Gillespie, Rencia

$48,49,49,51$

Grønbeck, Sturle

Gürkan, Aysel
52

45

\section{Page}

52

45

42

54

49

41

47

52

52

41

\section{2}

51

51,54

53

46

53

$48,49,49$

$40,46,46$

43,47

45

51

45

41

53

46

44,51

51

44

41

52

54

46

48

46

41
H

Harris, Candice

Hartley, Tina

Herbert, Vivien

Hiekkanen, Sofia

Hodkinson, Peter

Holmes, Nicky

Hoogendoom, Marga

Iliodromitis, EK

49

Isik, Mehmet

42

J

Jordan, Portia

$\mathrm{K}$

Kang, Jiyeon

Karabinis, Andreas

44,44

Karageorgou, Katerina

48,49

Karanikola, Maria

Karra, Vassiliki

Karyoti, Vassiliki

Kaymak, Isik

Kearns, Irene J

Keskin, Gulbahar

Khoza, Thembekile

Kil, YounKyoung

Kim, Eunsook

Kim, HyangSook

Kim, JungYeon

Kim, SangHee

Kivanc, Meral Madenoglu

Page

56

46

56

42

52

52

51

Kletsiou, Eleni

Ko, Hyun-Young

Kong, Yoke Kuan

Kotze, Renee

Kouskouni, Evaggelia

Kuan, Kong Yoke

Kum, Lee Siew

Kwon, Eunok

54

49

51

54

40

47

41

55

50

50

50

50

50

42,46

49

44

41

54

54

47

47

41

L

Langvad, Anne $\quad 46$

Lee, Ang Noi $\quad 41,42$

Lee, Eunjung $\quad 50$

Lee, EunSook $\quad 50$

Lee, Geummoon $\quad 50$

Lee, IIHak $\quad 50$

Lee, Jiyoon $\quad 50$

Lee, Siew Kum $\quad 41$

Lee, Yu-nah $\quad 50$

Lemonidou, Chrysoula $\quad 51$ 


\begin{tabular}{|c|c|c|c|}
\hline & Page & $\mathbf{R}$ & Page \\
\hline Leonard, Angela & 51 & Rageb, Om & 51 \\
\hline Lerdal, Anners & 45 & Randen, Irene & 45 \\
\hline Lien, Tine & 45 & Reyneke, Maryn & 51,54 \\
\hline Logothetis, Elias & 54 & Ristola, Elina & 42 \\
\hline Lucia, Sizakele & 55 & Rothgart, Maria & 46 \\
\hline M & & $\mathbf{S}$ & \\
\hline Manolidou, Zacharoula & 54 & Samuels, Hailey & 50 \\
\hline Matlakala, Mokgadi & 53 & Santoro, Cristiane & 51 \\
\hline Matlala, Benga Sidwell & 45 & Schmollgruber, Shelley & 48,56 \\
\hline Mehta, Ram Sharan & 43,43 & Scholtz, Suegnet & 40,55 \\
\hline Middleton, Nicos & 48,49 & Scribante, Juan & 53 \\
\hline Mitchell, Marion & 45 & Seow, Meow Kui & 41 \\
\hline Miranda, Dinis Reis & 51 & Shum, David & 45 \\
\hline Morrow, Brenda & 52 & Skafte, Anne & 46 \\
\hline Mpoti, Sofia & $48,49,49$ & Solms, Diana & 48 \\
\hline Mpouzika, Meropi & $48,49,49$ & Sousa, Regina & 51 \\
\hline Munyiginya, Paul & 47 & Stafseth, Siv K & $45,48,48,51$ \\
\hline Myburgh, Chris & 40,55 & $\mathbf{T}$ & \\
\hline $\mathbf{N}$ & & Turkoglu, Meryem & 42 \\
\hline $\mathrm{Na}$, Sun Gyoung & 44 & u & \\
\hline Nel, Elzabe & $40,45,55$ & 0 & \\
\hline Neo, Siew Cheng & 41 & Ufashingabire, Christine & 43 \\
\hline Noi, Lee Ang & 47 & Ulupınar, Sevim & 46 \\
\hline Nsereko, Etienne & 43 & Uysal, Ayse Hale & 41 \\
\hline Nyamakura, Rudo & 53 & V & \\
\hline 0 & & Venkatesh, Bala & 45 \\
\hline Odunaiya, Nse & 52 & $\mathbf{W}$ & \\
\hline Oh, Soonja & 50 & Wallis, Lee & 52 \\
\hline Ören, Besey & $40,41,46$ & Ward, Alison & 52 \\
\hline $\mathbf{P}$ & & Weisbrodt, Leonie & 50 \\
\hline Padilha, Katia & 51 & Wetzig, Sharon & 45 \\
\hline Pang, Nguk Lan & 41 & $\mathbf{Y}$ & \\
\hline Papanikolaou, Spiros & 54 & Yazici, Saadet & 40,41 \\
\hline Papathanassoglou, Elizabeth & $48,49,49,49,51$ & Yi, Young Hee & $44,46,50$ \\
\hline $\begin{array}{l}\text { Park, So Hyun } \\
\text { Perrie, Helen }\end{array}$ & $\begin{array}{l}50 \\
53\end{array}$ & Yildiz, Nebahat & 41 \\
\hline Petropoulou, Chrysa & 54 & Young, S Ann & 48 \\
\hline Phillips, Maria & $54,54,55,55$ & $\mathbf{Z}$ & \\
\hline Poggenpoel, Marie & 40,55 & Zengin, Neriman & 40,46 \\
\hline Pretorius, Ronel & 51,54 & & \\
\hline Pyo, MiYeon & 50 & & \\
\hline
\end{tabular}

